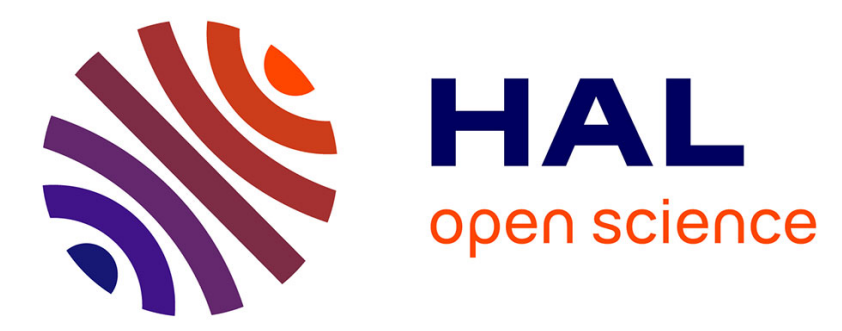

\title{
Experimental Investigations on Common Research Model at ONERA-S1MA-Drag Prediction Workshop Numerical Results
}

\author{
A. Cartieri, D. Hue, Q. Chanzy, O. Atinault
}

\section{- To cite this version:}

A. Cartieri, D. Hue, Q. Chanzy, O. Atinault. Experimental Investigations on Common Research Model at ONERA-S1MA-Drag Prediction Workshop Numerical Results. Journal of Aircraft, 2017, 10.2514/1.C034414 . hal-01570167

\section{HAL Id: hal-01570167 \\ https://hal.science/hal-01570167}

Submitted on 10 Oct 2018

HAL is a multi-disciplinary open access archive for the deposit and dissemination of scientific research documents, whether they are published or not. The documents may come from teaching and research institutions in France or abroad, or from public or private research centers.
L'archive ouverte pluridisciplinaire HAL, est destinée au dépôt et à la diffusion de documents scientifiques de niveau recherche, publiés ou non, émanant des établissements d'enseignement et de recherche français ou étrangers, des laboratoires publics ou privés. 


\title{
Experimental Investigations on the CRM at ONERA-S1MA - Drag Prediction Workshop Numerical Results
}

\author{
Aurelia CARTIERI ${ }^{1}$, David HUE ${ }^{2}$, Quentin CHANZY $^{3}$, and Olivier ATINAULT ${ }^{4}$ \\ ONERA-The French Aerospace Lab, 91120 Palaiseau, France
}

\begin{abstract}
This paper aims at presenting some of the experimental and numerical results obtained with the NASA-Boeing Common Research Model at ONERA. The wind tunnel model used in the present study is the ONERA Large Reference Model (1/16.835) which has the same geometry as the CRM considered in the latest AIAA Drag Prediction Workshops. Experimental data has been collected from the ONERA-S1MA wind tunnel at Mach numbers between 0.30 and 0.95 and a mean aerodynamic chord Reynolds number of 5 million for four different configurations: wing-body only and wing-body with horizontal and/or vertical tails. Force and moment, pressure and surface flow measurements have been performed. Concerning the numerical study, all the RANS computations have been completed with the structured solver elsA, the Spalart-Allmaras and kw-SST turbulence models have been used as well as the Quadratic Constitutive Relation. In this paper, configuration effects (increments due to horizontal and/or vertical tails) are assessed both numerically and experimentally for several Mach numbers and angles of attack. The delicate issue of flow separation at wing-body junction is also addressed with the support of oil flow visualizations. elsA and S1MA skin pressure distributions are presented; the agreement is satisfactory except for some outboard wing sections at high lift levels. Finally, comparisons of drag and moment values including CFD and test data from different wind tunnels are proposed (S1, NASA Ames and NTF, and ETW).
\end{abstract}

\section{Nomenclature}

$$
\begin{array}{ll}
\text { Alpha } & =\text { angle of attack } \\
A R & =\text { aspect ratio } \\
b & =\text { aircraft span } \\
C D & =\text { near-field drag coefficient } \\
C D f & =\text { friction drag coefficient }
\end{array}
$$

\footnotetext{
${ }^{1}$ Engineer, Wind Tunnel Division, aurelia.cartieri@onera.fr AIAA Member.

${ }^{2}$ Engineer, Applied Aerodynamics Department, david.hue@onera.fr, AIAA Member.

${ }^{3}$ Engineer, Applied Aerodynamics Department, quentin.chanzy@onera.fr

${ }^{4}$ Engineer, Applied Aerodynamics Department, olivier.atinault@onera.fr
} 


$$
\begin{array}{ll}
C D p & =\text { pressure drag coefficient } \\
C L & =\text { lift coefficient } \\
C M & =\text { pitching moment coefficient } \\
C p & =\text { pressure coefficient } \\
M a & =\text { Mach number } \\
p & =\text { static pressure } \\
\text { Re } & =\text { Reynolds number } \\
\text { Sref } & =\text { reference surface area } \\
\text { Lref } & =\text { reference length } \\
u, v, w & =\mathrm{x}, \mathrm{y}, \mathrm{z} \text { velocity components } \\
Y^{+} & =\text {normalized first cell height } \\
\eta & =\text { fraction of wing span } \\
\infty & =\text { subscript for freestream state value } \\
\mathrm{HTP} & =\text { Horizontal Tail Plane } \\
\mathrm{VTP} & =\text { Vertical Tail Plane } \\
\mathrm{WB} & =\text { Wing-Body } \\
\mathrm{WBH} & =\text { Wing-Body with HTP } \\
\mathrm{WBV} & =\text { Wing-Body with VTP } \\
\mathrm{WBVH} & =\text { Wing-Body with VTP and HTP }
\end{array}
$$

\section{Introduction}

$\mathrm{T}_{\mathrm{w}}^{\mathrm{H}}$ HE Common Research Model (CRM) developed by NASA and Boeing [1,2] serves as a reference for providing wind tunnel data aimed at the validation of codes dedicated to aircraft performance prediction. This model has been designed and built as part of the AIAA Drag Prediction Workshop (DPW) series [3]. For many years now, ONERA has participated to these fruitful workshops $[4,5,6,7]$ thus enhancing its experience in the field of aircraft performance assessment through numerical approaches. Recently, ONERA has built its own CRM model; it is the Large Reference Model (LRM). It has been designed to have the same geometry as the NASA model when submitted to equivalent constraints. The ONERA model will be used to verify the complete measurement chain of the SIMA facility [8], which is one of the greatest transonic wind tunnels in the world. These verifications will concern the wind tunnel structure itself (for instance after repairs and modifications), quality of the airstream, checkouts of data repeatability over time. Moreover, in the meantime, this model is also used for technical development of new measurement techniques and devices as well as a reference for Computational Fluid Dynamics (CFD) validation at ONERA.

At the end of 2014, the first test campaign involving the LRM took place in S1MA. The main purpose of this test was to acquire a large reference database on this model. Measurements such as force and moment quantification (balance), dynamic and static skin pressure probing, and surface flow visualization have been performed for large ranges of Mach numbers (0.30 to 0.95$)$ and lift levels. Four configurations of the model have been assessed in the 
wind tunnel: the Wing-Body only (WB), the WB with Horizontal Tail Plane (WBH), the WB with Vertical Tail Plane (WBV), and finally the WB with both HTP and VTP (WBVH). The VTP of LRM has been designed by ONERA so that the reference model of S1MA can be used for tail increment evaluation; this geometry has been shared with the community and is presented in [9].

More recently, using the geometries and meshes provided in the framework of DPW-6 held in 2016, new CFD studies have been completed at ONERA to carry out comparisons with the test data obtained in S1MA. These computations have been performed using structured Overset grids with the RANS solver elsA.

In order to highlight the results of these complementary experimental and numerical activities, the paper will be organized as follows: first, the S1MA wind tunnel and the LRM model will be described as well as the test campaign of 2014. Concerning the CFD aspects, the grids and solver settings (including the turbulence models) will be presented. Thereafter, the wind tunnel and numerical results will be given and compared, starting with a study of increments due to the horizontal and vertical tailplanes. Then, the WB junction flow separation issue will be analysed. Finally, comparisons will be carried out on the wing pressure distributions as well as the drag and moment predictions; for this matter, CRM data from other wind tunnels such as the NASA National Transonic Facility (NTF), Ames, and also the European Transonic Wind tunnel (ETW) [2,10] are introduced.

\section{Wind Tunnel Tests}

\section{A. Facility Description}

S1MA is a continuous atmospheric wind tunnel operating in the sub/transonic regime. It was put into service in 1952 and is equipped with two contra-rotating fans, driven by Pelton turbines, the power of which is 88 MW. The wind velocity can be varied from a few meters per second to approximately Mach 1 by varying the fan speed. The total length of the aerodynamic circuit is about $400 \mathrm{~m}$ (see Fig. 1). The test section dimensions are $14 \mathrm{~m}$ in length and $8 \mathrm{~m}$ in diameter. For a Mach number around 0.85, the Reynolds number per meter is about 11 million.

A peculiarity of the circuit is the absence of heat exchanger. The temperature is controlled by letting outside fresh air enter the circuit. Hot air naturally exhausts around the edge of the contraction through an annular exit. An exhaust rate of about $10 \%$ of the total mass flow is required to maintain a temperature of about $50^{\circ} \mathrm{C}$ in the tunnel.

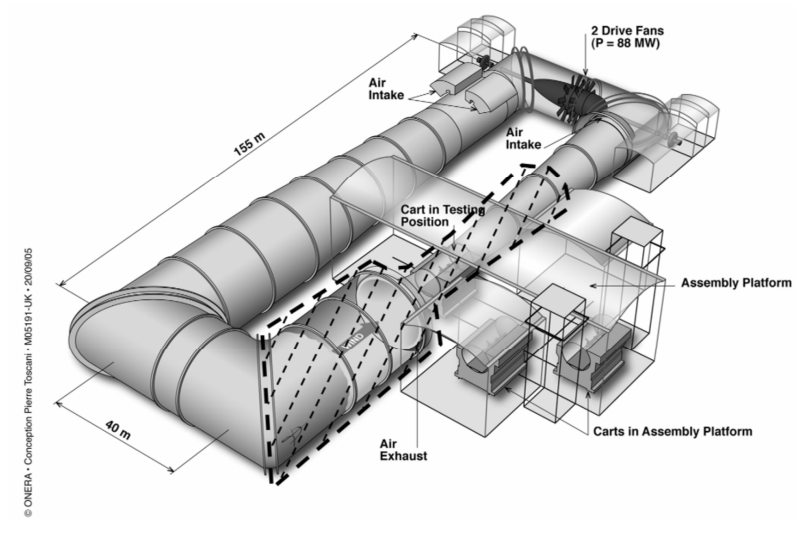

Fig. 1 S1MA air circuit. 


\section{B. Model description}

The model used in the current investigation is the ONERA Large Reference Model (LRM) which has the same geometry as the CRM. It was sized to $220 \%$ of the NASA model, so the final scale is $1 / 16.835$. This configuration consists of a contemporary supercritical transonic wing $(A R=9.0)$ and a fuselage that are representative of a widebody commercial transport aircraft. The vertical tail geometry was designed by the Civil Aircraft Unit of ONERA in cooperation with the Wind Tunnel Division.

The CRM is designed for a cruise Mach number of 0.85 and a corresponding lift coefficient of $C L=0.5$. The S1 model is defined by mean aerodynamic chord $L r e f=0.4161 \mathrm{~m}$, reference surface area $S r e f=1.3538 \mathrm{~m}^{2}$ and a span $b$ $=3.4905 \mathrm{~m}$.

The ONERA LRM model was designed so that it has the same deformation at cruise point as the CRM model tested in the NTF Wind Tunnel. The main dimensions of the model are given in Fig. 2.

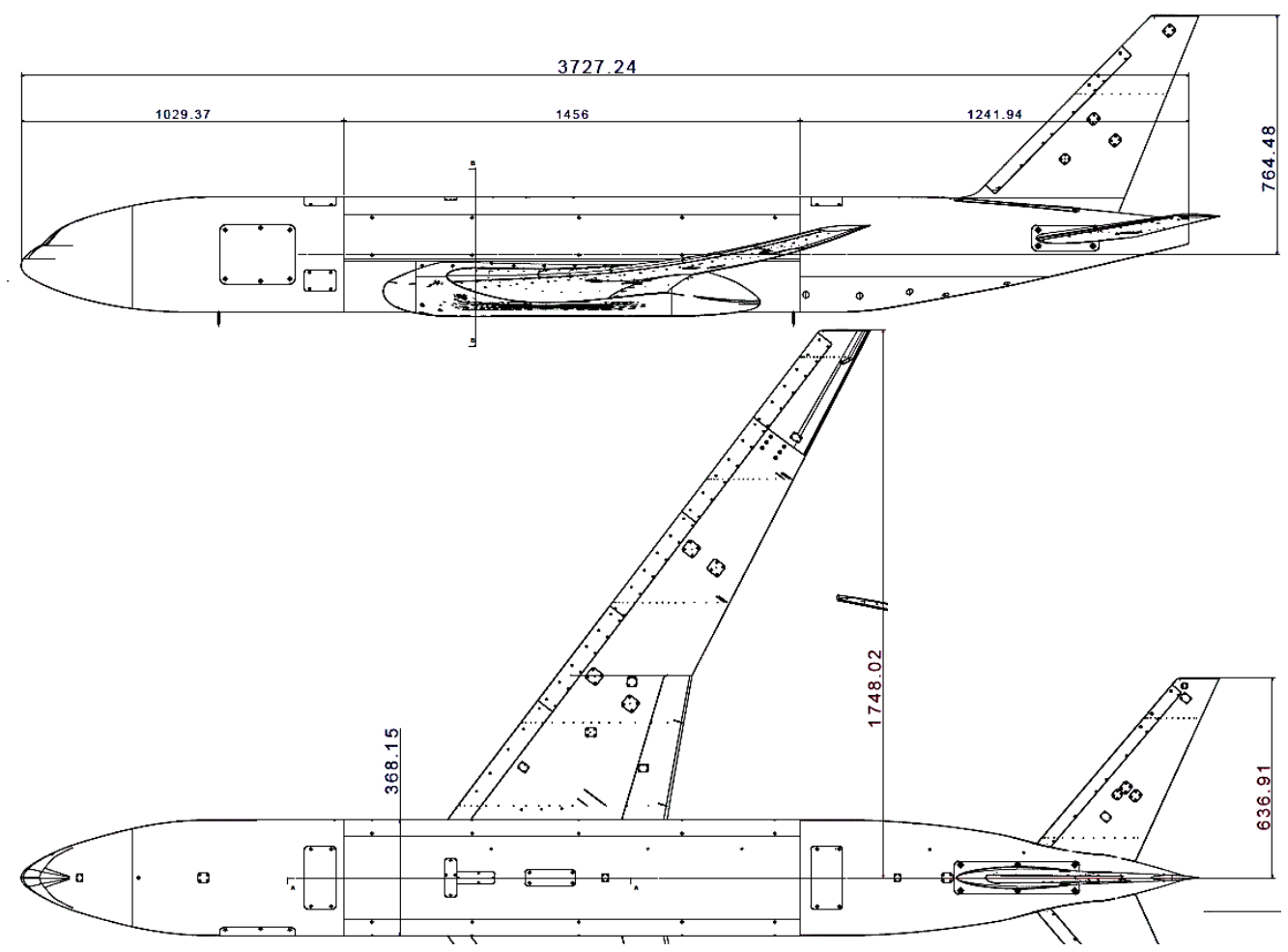

Fig. 2 Main dimensions of the LRM model.

Pressure distributions are measured on both the left and right wings using 270 pressure orifices located in 9 spanwise stations (the same as the NASA CRM wind tunnel model) $: 5$ on the right wing $(\eta=0.131,0.283,0.502$, 0.727 and 0.950$)$ and 4 on the left wing $(\eta=0.201,0.397,0.727$ and 0.846$)$. There were also one section on the VTP, and three sections on the HTP $(\eta=0.2,0.5$ and 0.8$)$. The fuselage was also equipped as shown in Fig. 3. 


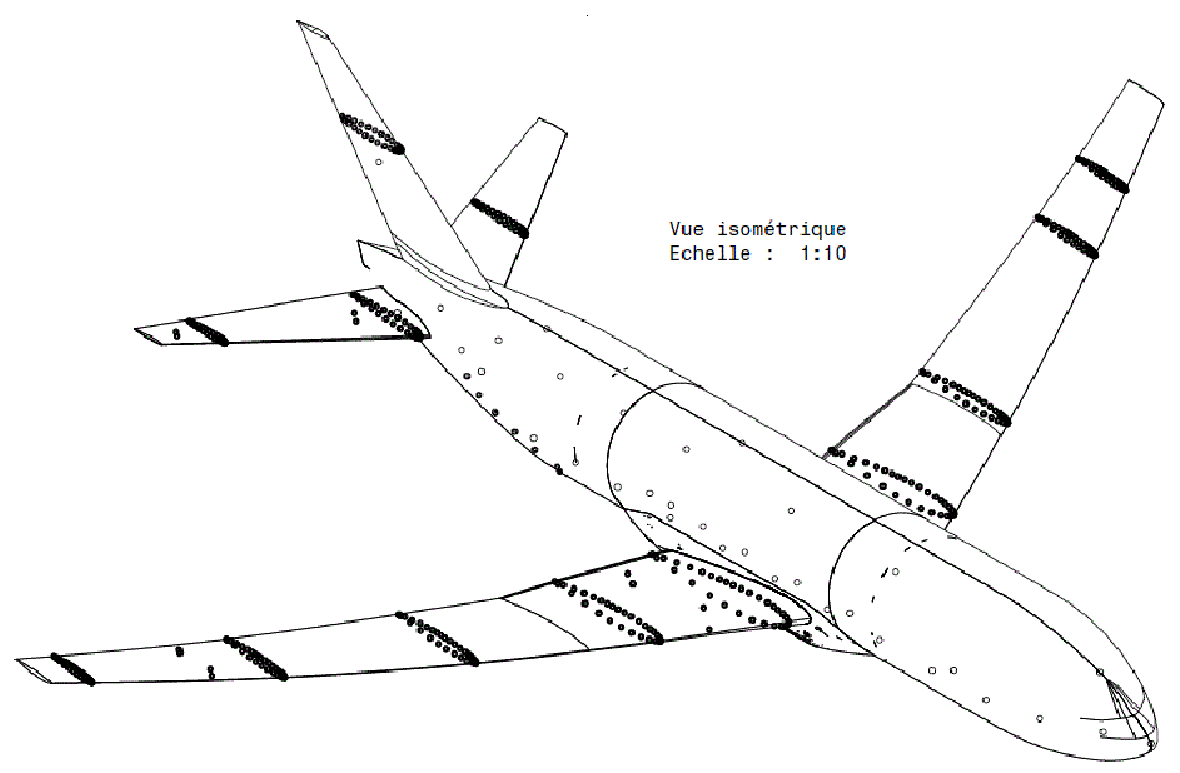

Fig. 3 Pressure measurements.

All pressure measurements were made using Electronically Scanned Pressure (ESP) modules installed inside the forward portion of the fuselage. The model is mounted in the wind tunnel using a Z sting setup as shown in Fig. 4.
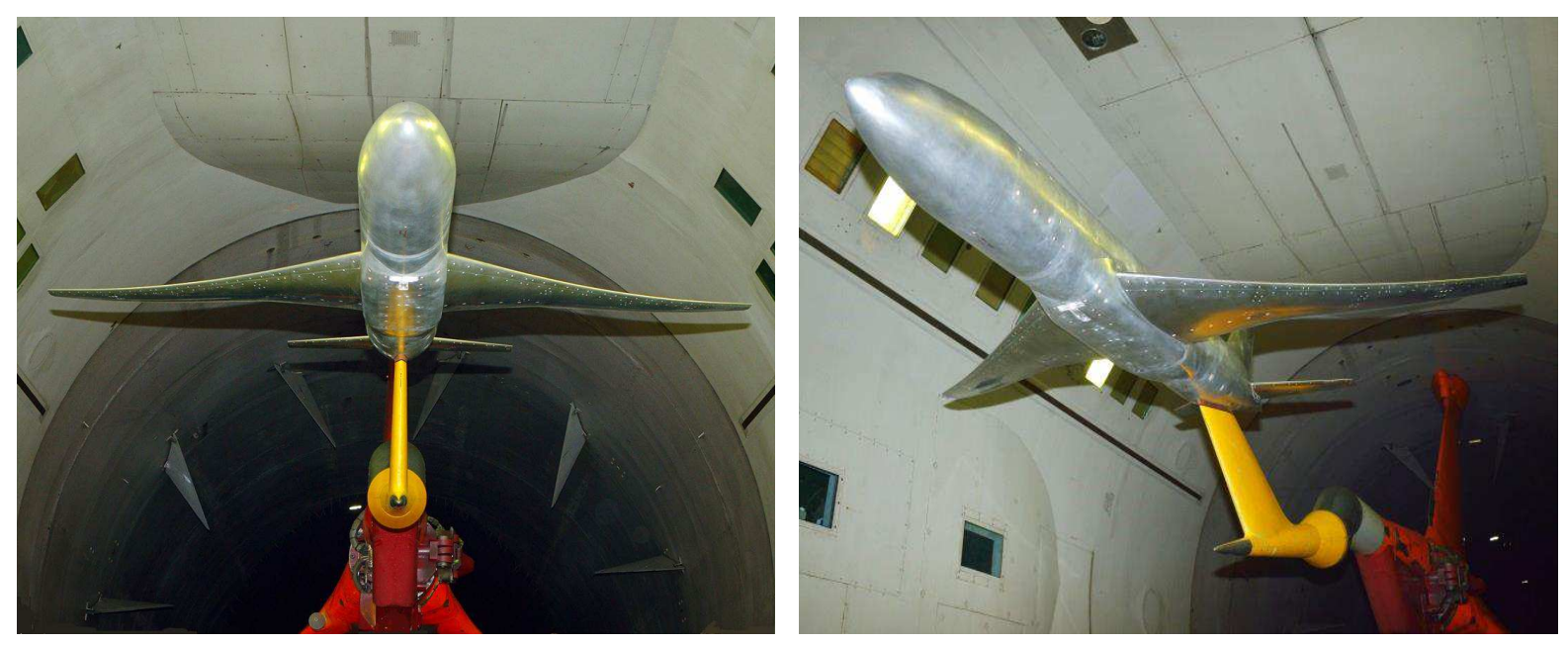

Fig. 4 The LRM model in S1MA wind tunnel.

\section{Test conditions and measurements}

The tests were carried out in a Mach number range going from $M a=0.30$ up to $M a=0.95$. The Reynolds number based on mean aerodynamic chord was 5 million. The incidence range was from $-3.0^{\circ}$ to $+10.0^{\circ}$. The incidence of the model was measured by means of three goniometers connected to the weighed balance adapter. It was corrected for wall and sting effects (see below) and for the wind tunnel up-wash that was determined during the 
campaign. The forces and moments of the model were measured with a six-component balance equipped with two temperature sensors. The wing deformation measurements were performed with two high resolution cameras located in the ceiling of the test section [11] behind a window. The bending and twist deformations of the right wing were derived from the comparison of the 3D target positions between wind-on and wind-off conditions. The twist measurements obtained at $M a=0.85$ and $C L=0.5$ were in good agreement with the results obtained in NTF and ETW wind tunnels.

The transition of the boundary layer on the different parts of the model was forced by means of Cadcut strips. A Cadcut strip consists of an adhesive band on which dots (measuring $1.3 \mathrm{~mm}$ in diameter) are precut by laser at regular intervals (spaced $2.4 \mathrm{~mm}$ apart). The trips dots were installed at $10 \%$ chord on the wings, the HTP and the VTP. The trip dots were $0.142 \mathrm{~mm}$ high on the wing and $0.127 \mathrm{~mm}$ high on the tails. On the fuselage, the trips were applied at $60 \mathrm{~mm}$ from the nose and measured $0.152 \mathrm{~mm}$. Some acenaphtene visualizations were performed at the beginning of the test campaign in order to check the effectiveness of the boundary layer tripping.

Finally, some colored oil flow visualizations were also performed.

\section{Correction Methods}

The aerodynamic interferences are taken into account thanks to a correction process composed of several contributions:

1) The empty test section correction: it is a Mach number correction that results from a test section tunnel calibration;

2) The buoyancy correction: it is the effect of the empty wind tunnel Mach number gradient on drag (which is proportional to the product of the gradient and the effective volume of the body);

3) The wall effect correction: these corrections rely on the potential flow theory [12]. Under the assumption that the flow in the tunnel is irrotational outside the boundary layers and wakes, it can be described by a velocity potential $U_{0} x+\varphi$. Assuming now that the velocity perturbations $\partial x \varphi, \partial y \varphi$ and $\partial z \varphi$ are small with regard to $U_{\infty}$, one comes to the well-known linearized potential equation:

$$
\left(1-M a_{\infty}^{2}\right) \partial_{x}^{2} \varphi+\partial_{y}^{2} \varphi+\partial_{z}^{2} \varphi=0
$$

with boundary conditions at solid walls linearized as well.

Unfortunately, this last assumption is less and less valid as the upstream Mach number $M a_{\infty}$ values approach $M a=1.00$ and as typical transonic phenomena occur on the model, with large fluid accelerations up to supersonic regime.

This equation and the corresponding boundary conditions can be solved through a distribution of singularities on the model and support. The intensity of each singularity is based on the cross section areas, the lift and the drag. 
Once the proper singularities have been set up, the linearity of Eq. (1) allows the potential $\varphi$ to be broken down into a field $\varphi m$ generated by the model and a field $\varphi s$ generated by the support. Hence $\nabla \varphi s=(u s, v s$, $w s)$ is the field of velocity distortion generated by the support.

Once the velocity field $\nabla \varphi s$ is known, one can easily determine a field of Mach number distortion:

$$
\delta M a=M a_{\infty}\left(1+\frac{\gamma-1}{2} M a_{\infty}^{2}\right) \frac{u_{s}}{U_{\infty}}(2)
$$

and a field of angle of attack distortion (upwash):

$$
\delta \text { Alpha }=\frac{w_{s}}{U_{\infty}}(\mathbf{3})
$$

These fields are then averaged in space over areas of aerodynamic significance.

The Mach number correction $\Delta M a$ is taken as the value of $\delta M a$ at $1 / 4$ of mean aerodynamic chord. The alpha correction is computed from a slightly more elaborated process: it is chord-averaged along the wing span, at $3 / 4$ of local chord, this correction enabling the lift correction to be zero (theory of Pistolesi, [13]).

Second order corrections on drag (buoyancy correction due to velocity distortion) and pitching moment (mainly due to the HTP lift gradient to alpha) are then calculated;

4) The sting corrections: these corrections are calculated thanks to RANS computations [14]. First order corrections are determined thanks to a pairing process. With and without support simulations are considered as paired when the flow fields around the wing are similar. The criterion of similarity is the RMS of pressure coefficient distortion on the wing. The corrections to forces and moments are deduced from the differences between the integrated forces over the model with and without support.

5) The sting cavity pressure correction: this correction results from the presence of a pressure coefficient (not zero) inside the rear fuselage which is "open" to enable sting entry. It consists in replacing, on the cavity surface, the mean measured cavity pressure by the reference pressure.

\section{Numerical Simulations}

\section{A. Geometry and Grids}

The wing geometries used for the numerical computations of this study are the ones proposed in the recent DPW-6. They are at scale 1/1 (real aircraft dimensions). The original DPW-5 wing geometry did not match the experimental shape. When the model was tested in the wind tunnel, the wings twisted more than the one used for the computational analysis [15]. For DPW-6, the twist and bending data obtained during the CRM campaign in the European Transonic Wind tunnel have been used to generate wing geometries corresponding to different angles of attack as shown in Fig. 5. The aero-elastic effects are therefore taken into account. 
To perform the RANS computations on these geometries, the meshes "Overset grids Boeing Serrano.REV00" of DPW-6 (available on the DPW website [3]) have been used. These structured Overset grids proposed by Boeing exhibit different refinement levels. They have been employed only for the WB configuration; the grids of horizontal and vertical tails are ONERA meshes presented further.

In Table 1 are presented the Boeing grid characteristics; the wing geometry is the one corresponding to an experimental angle of attack of $2.75^{\circ}$ and a lift coefficient of 0.5 and is therefore referenced as $2 \mathrm{p} 75$. Additional medium grids describe the appropriate wing geometries for four increasing angles of attack $(2 \mathrm{p} 50,3 \mathrm{p} 25,3 \mathrm{p} 75$, $4 \mathrm{p} 00)$.

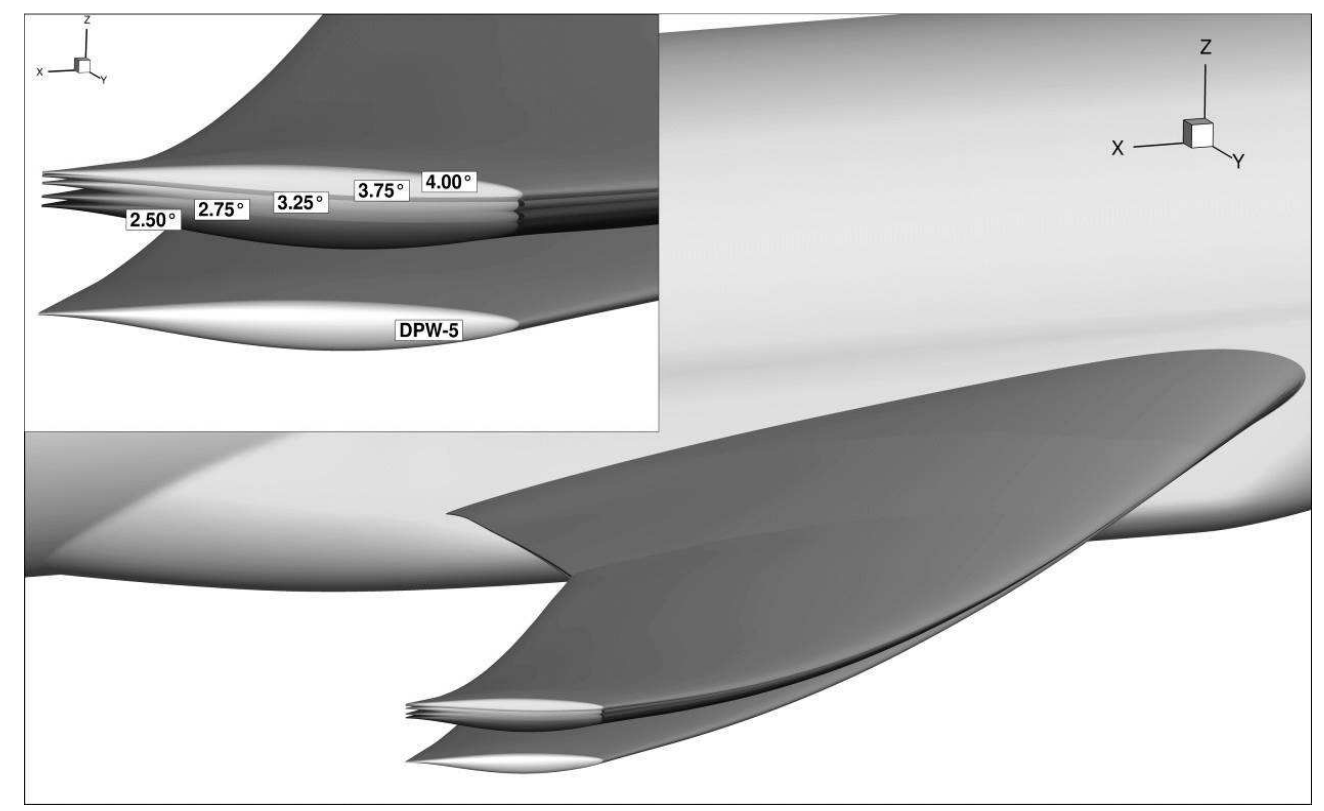

Fig. 5 CRM wing shapes at different angles of attack.

Table 1 “Overset grids Boeing Serrano.REV00” 2p75

\begin{tabular}{|c|c|c|c|}
\hline & & \multicolumn{2}{|c|}{ Wing-Body 2p75 } \\
\hline $\mathbf{n}^{\circ}$ & Level & millions of points $(\boldsymbol{N})$ & Average $\boldsymbol{Y +}$ \\
\hline $\mathbf{1}$ & Tiny & 7.4 & 0.78 \\
\hline $\mathbf{2}$ & Coarse & 14.4 & 0.59 \\
\hline $\mathbf{3}$ & Medium & 24.7 & 0.51 \\
\hline $\mathbf{4}$ & Fine & 39.1 & 0.44 \\
\hline $\mathbf{5}$ & Extra Fine & 58.2 & 0.38 \\
\hline
\end{tabular}


These Overset meshes are O-type grids created by extrusion of a surface discretization, as illustrated in Fig. 6, while the computational domain is described by three Cartesian boxes of decreasing refinement levels. The mesh extent is greater than 500 mean-aerodynamic chords. It can be also noticed in Fig. 6 with a slice in the wing mesh that the medium grids that will be largely used in this work exhibit a refinement level and a number of points that are already very satisfactory for RANS purposes.

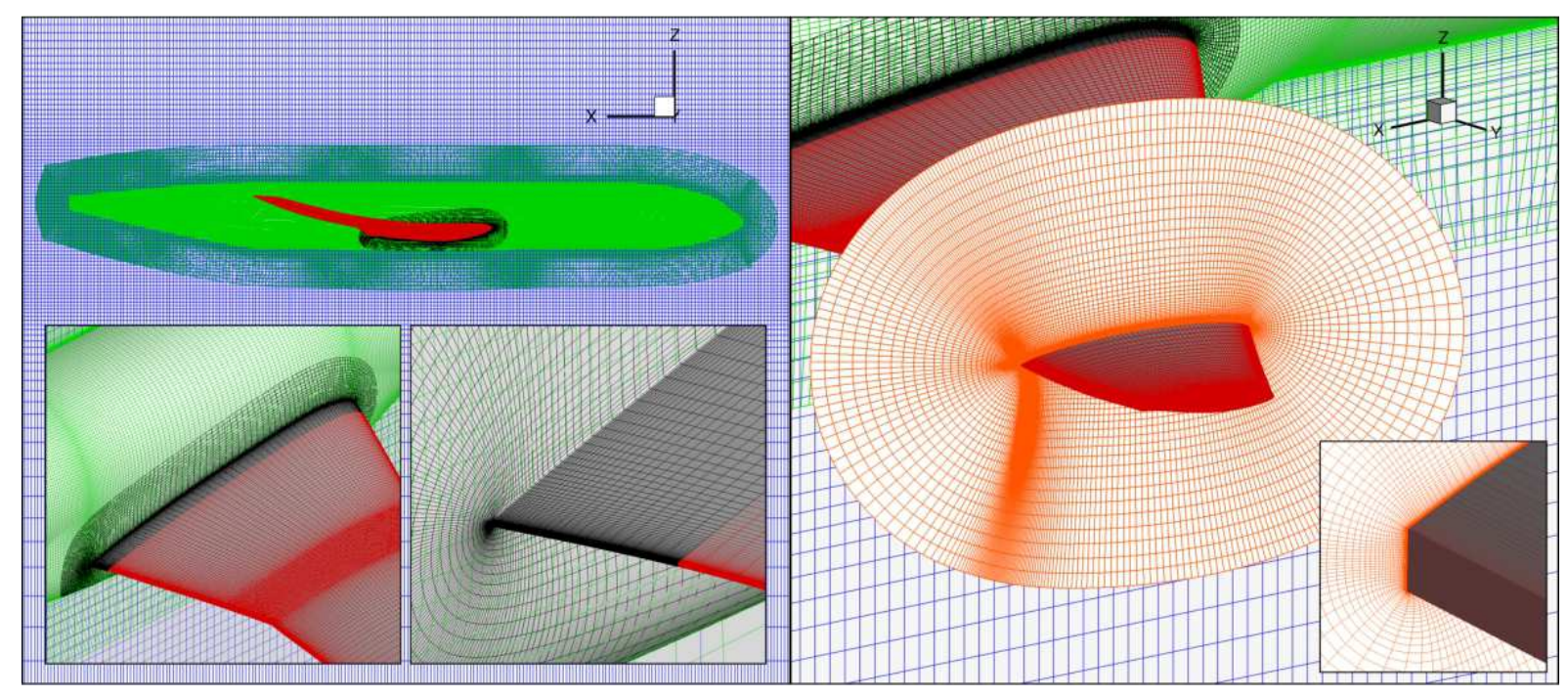

Fig. 6 Illustration of Boeing Overset grid topology (WB3).

Before running these grids from Boeing with the elsA solver, a necessary pre-processing has been handled. Eight different bases describe the WB configuration: three external boxes, the fuselage body and fuselage nose, the collar grid at wing-body junction, the wing itself, and the wing tip.

Along the whole process, the in-house software Cassiopee [16] has been extensively used. The blanking step which consists in removing all cells that are inside physical bodies has been realized using the latest Cassiopee blanking function. More detail can be found in [7].

In addition to the grids of the WB configuration presented above, the ONERA grids of the horizontal and vertical tails introduced in [9] have been used. It is an advantage of the Overset approach to allow different elements to be added or removed quite simply from an existing mesh. The tail grids have been realized with the software Pointwise [17] which allows satisfactory 3D grids to be generated from a surface discretization defined by the user (automatic extrusion). The HTP and VTP meshes are also basic O-type grids. The HTP mesh is made of about 1.8 million cells. It is shown in Fig. 7. In terms of topology and aspect, the VTP grid shape is equivalent. Nevertheless, the refinement level is lower for the VTP. It only includes 0.8 million elements. This is due to the fact that fewer aerodynamic interactions are expected in this area. Fig. 8 shows the complete mesh of the vertical tail. For sake of clarity, the cut at mid-chord is blanked after mid-span. 


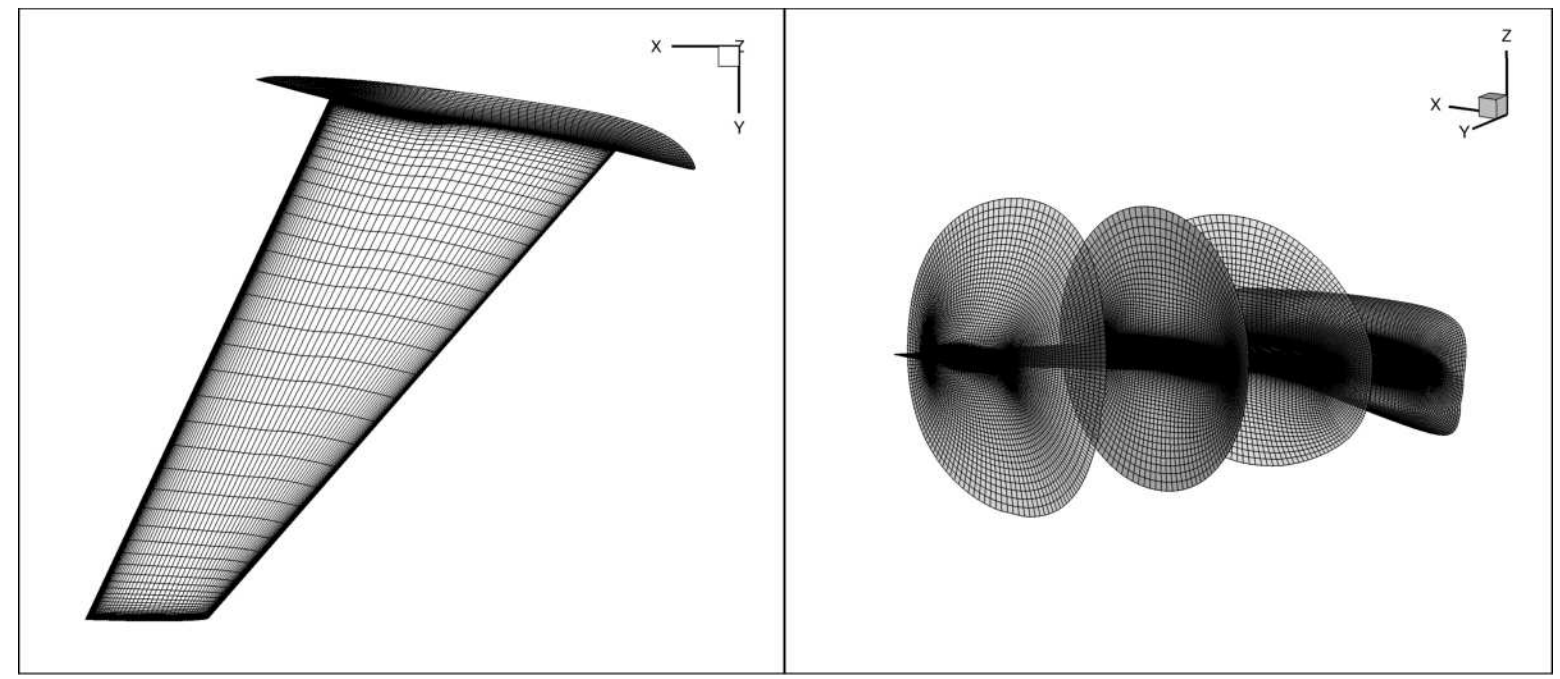

Fig. 7 HTP surface discretization and 3D mesh slices.

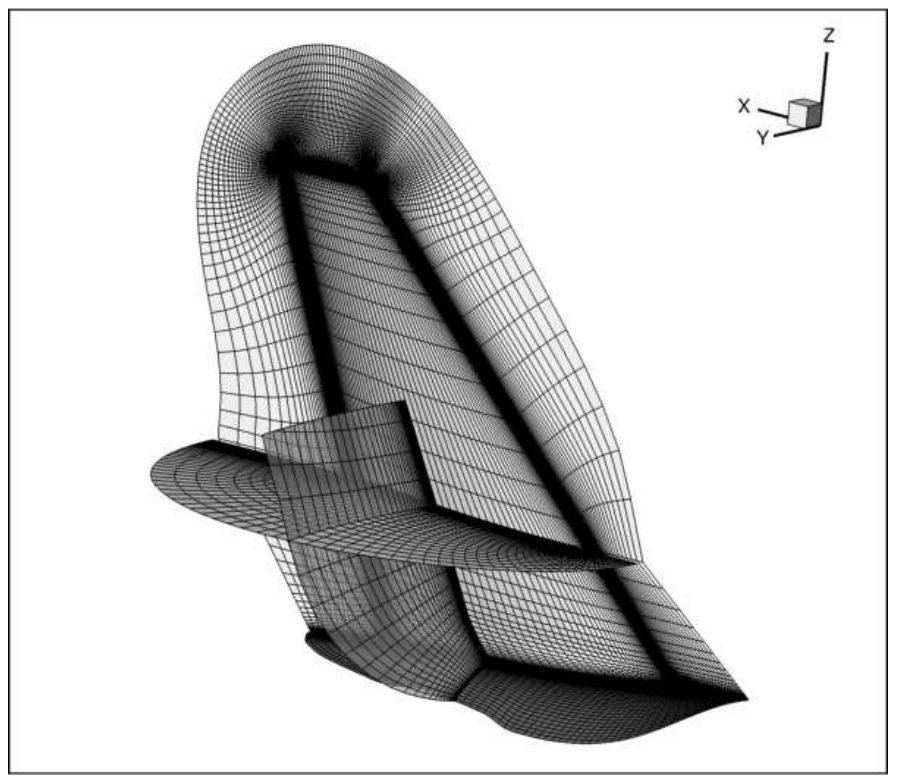

Fig. 8 VTP surface discretization and 3D mesh slices.

\section{B. ONERA - elsA RANS Solver}

As mentioned previously, all the computations have been performed with elsA [18]. This software uses a cellcentered finite-volume discretization on structured point-matched or Overset grids. In this study, time integration is carried out by a backward-Euler scheme with implicit LU-SSOR relaxation. Spatial discretization is realized using a $2^{\text {nd }}$ order central Jameson scheme [19] with scalar artificial viscosity. Multigrid techniques (one level) are used to accelerate convergence. 
Turbulence effects have been simulated with two different models: the one-equation Spalart-Allmaras model [20], and the two-equation k $\omega$-SST model of Menter [21]. For the latter, the limiter of Zheng [22] has been used as well as the dissipation wall treatment proposed by Wilcox in [23] where $\omega$ is a function depending on a pseudoroughness height $\mathrm{k}^{+}\left(\mathrm{k}^{+} \sim 5\right.$ for smooth walls). When specified, these models have been employed with the Quadratic Constitutive Relation (QCR) proposed in [24]; it leads to a nonlinear version of the model which does not use the traditional Boussinesq relation anymore.

The Overset interpolations are classically performed over two cell layers around holes and overlap conditions and a double-wall algorithm is used to ensure accurate interpolations when surfaces are described by several grids (collar grid for instance).

In order to reach a satisfactory level of convergence, the computations were continued at least until the fluxes were stable enough to observe a lift coefficient variation inferior to $+/-0.001$ and a drag coefficient variation inferior to one drag count over the last thousand of iterations $\left(1 \mathrm{drag}\right.$ count $\left.=10^{-4}\right)$. This is illustrated in Fig. 9 which exhibits the numerical convergence obtained with the medium grid WB3 as an example. A grid convergence study involving the grids described in Table 1 and computed with the elsA software is available in [7]: it demonstrated that the medium grid is fine enough to provide drag values almost identical to the ones obtained with the finest grid (less than 0.5 count of difference). The elsA simulations have been executed on a Silicon Graphics cluster (SGI ICE 8200) composed of 5,120 cores representing a power of 57.9 teraflops. The computations carried out for this work on the medium grids were performed in parallel mode using 48 or 96 cores: an 8000-iteration run typically took about 11 or 6 hours respectively.

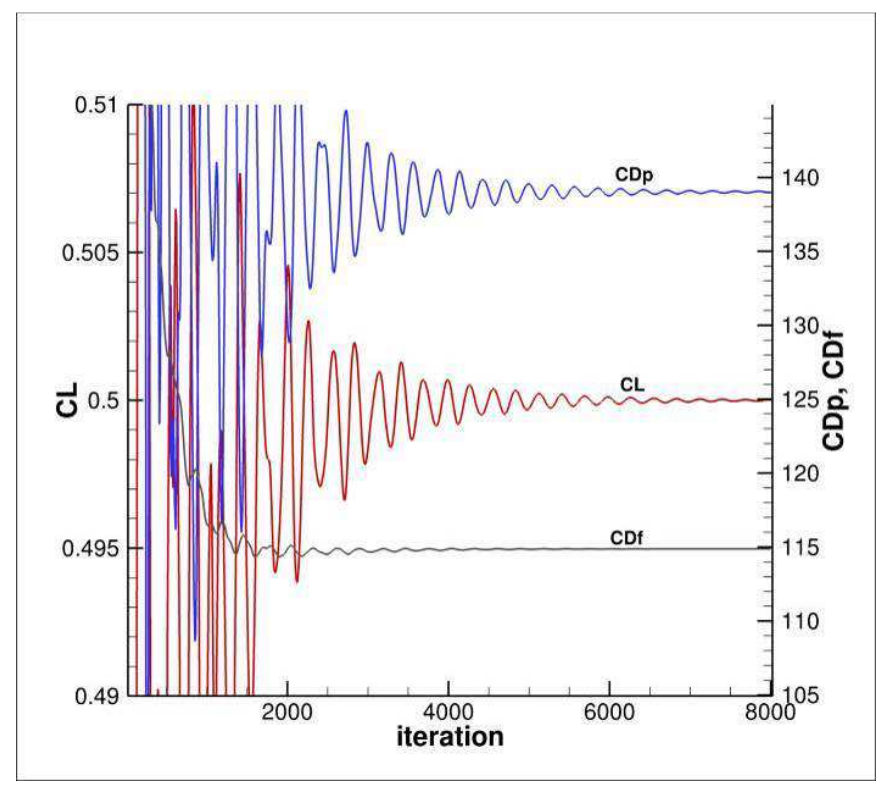

Fig. $9 M a=0.85, R e=5 \times 10^{6}, C L=0.5$; numerical convergence for the medium grid WB3. 


\section{Configuration Effects: Experimental/Numerical Comparison}

Determining the effects of adding HTP and/or VTP to the WB configuration was one of the purposes of this first test campaign. Indeed, the quantification of increments is of great interest in the current aeronautical industry. Concerning the associated numerical simulations, they have been performed at $M a=0.85$ only. All the geometries used are the ones of DPW-6 and the grids are the Overset grids presented in section III. For low lift levels, the wing geometry referenced as 2p50 was employed. For higher $C L$, the appropriate wing geometries were used (medium grids). Turbulence has been simulated with the one-equation Spalart-Allmaras model and the Quadratic Constitutive Relation. Transition was fixed for all wind tunnel tests whereas the computations are fully turbulent.

\section{A. HTP effect without VTP}

The HTP effect without VTP is first investigated. Fig. 10 shows the experimental effects at $M a=0.85$. The addition of the horizontal tailplane increases the drag, lowers the lift and significantly increases the pitching moment.

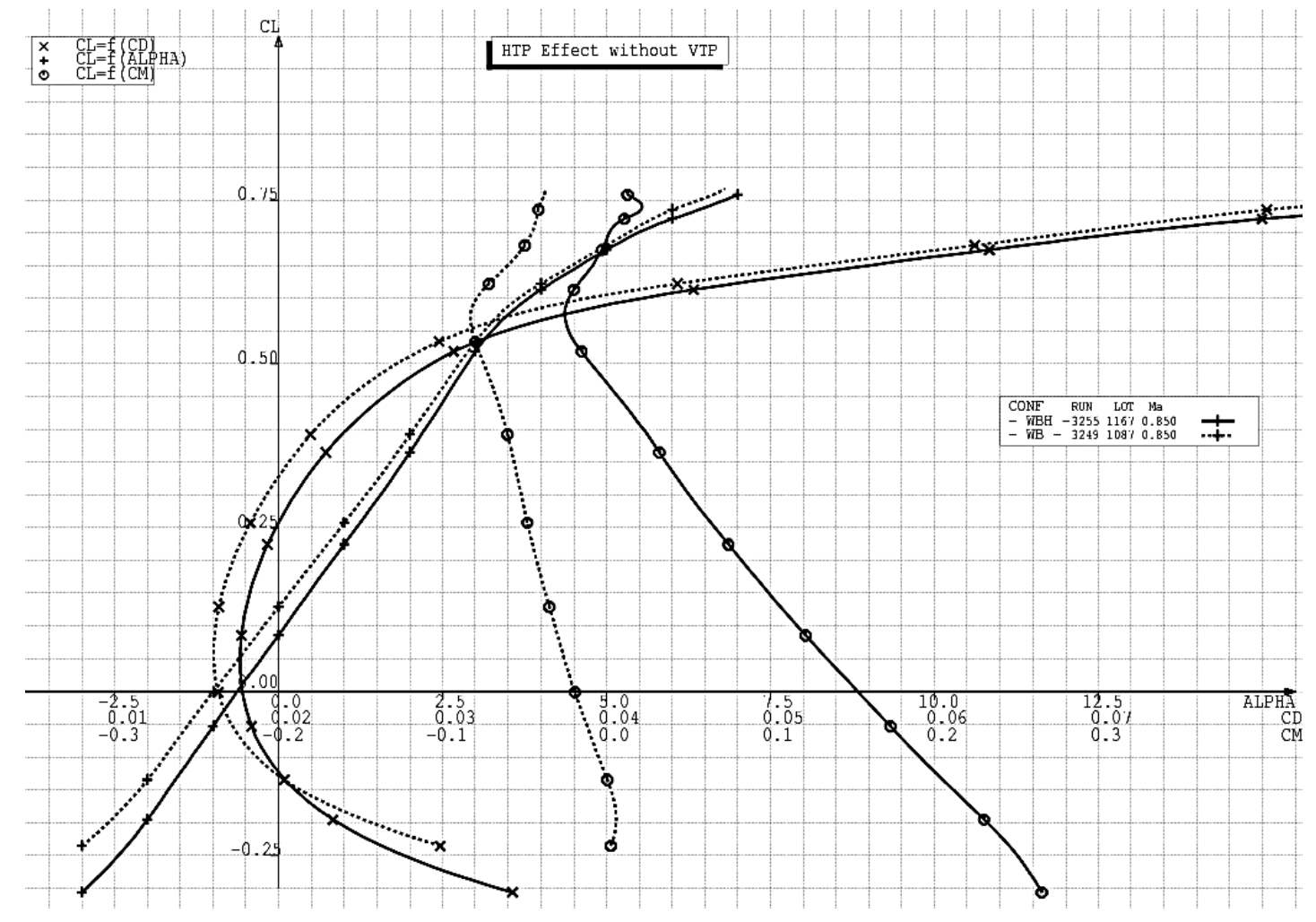

Fig. 10 Experimental HTP effect at $M a=0.85$ and $C L=0.5$.

The effect is presented in increments (at constant lift) in Fig. 11 for Mach number values of 0.7, 0.85 and 0.87. The Alpha increase reduces with lift. The drag increase is constant up to $C L=0.5$, then increases for higher $C L$. The pitching moment effect is dependent on $C L$ and varies with Mach number. For $C L=0.5$ and $M a=0.85$, the horizontal tailplane effect is about $0.15^{\circ}$ of Alpha, 20 drag counts (d.c.) and 0.07 of pitching moment. The agreement between CFD and wind tunnel data is good in the range of lift levels numerically investigated. 
The HTP generates negative lift over the whole polar, and therefore a positive (nose-up) pitching moment as expected. The DCM*Lref/DCL ratio is consistent with the HTP location. It gives a distance of $25 \mathrm{~m}$ (which is the distance between the model reference center and the HTP) at scale 1/1 for a lift variation of 0.02 and a pitching moment variation of 0.07 . The HTP effect on the near-field drag coefficients is shown in Fig. 12. The impact on friction drag represents about 12 d.c. and is constant with lift. This increment is consistent with an increase of $10 \%$ of the wetted surface area. The effect on the pressure drag increases with $C L$.
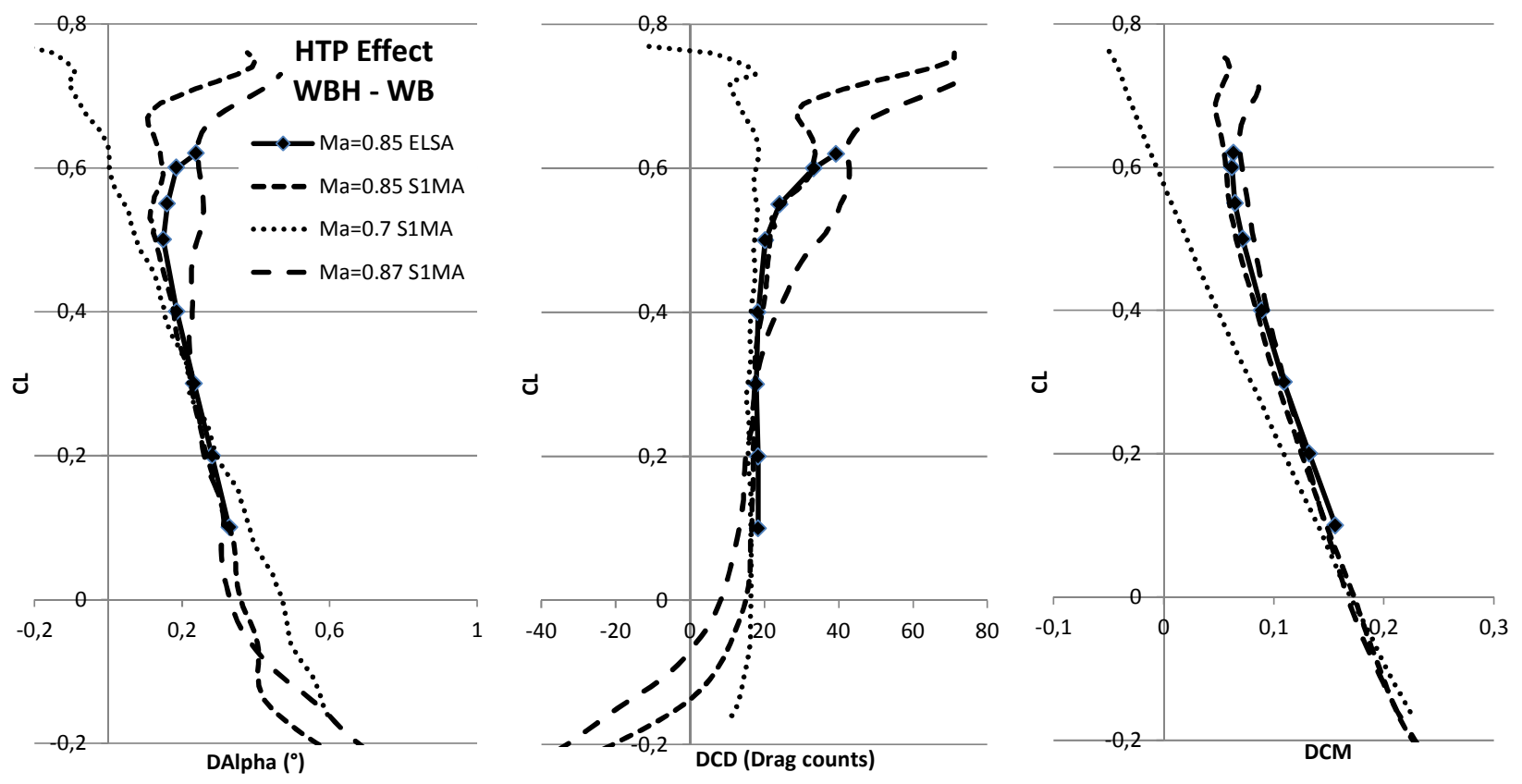

Fig. 11 Numerical and experimental HTP effect (WBH minus WB). 


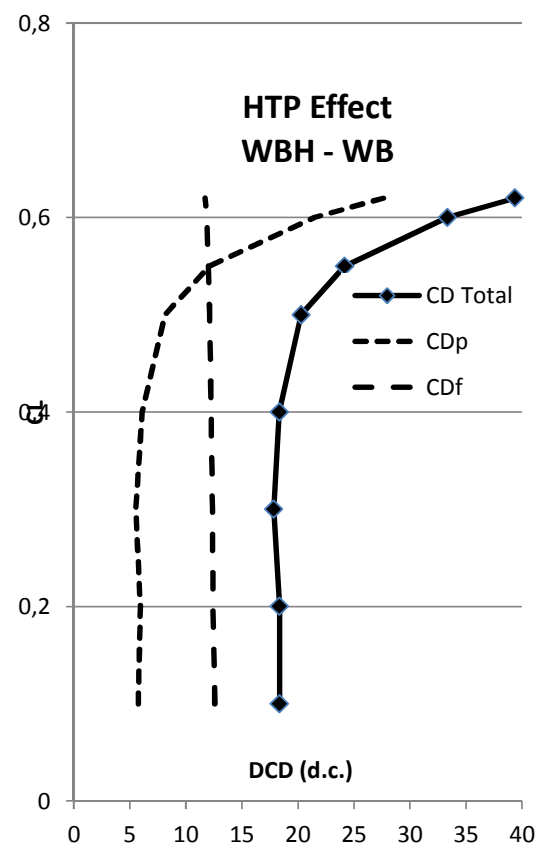

Fig. 12 Numerical HTP effect on near field drag at $M a=0.85$.

Flow distortion generated by the HTP can be observed in Fig. 13 that displays pressure coefficient disturbance on the model skin at $C L=0.5$. Experimental results are added to numerical results (spheres indicates pressure taps colored by experimental results). The larger disturbances are logically observed closed to the HTP. The front fuselage is not affected by the horizontal tailplane. The agreement between numerical and experimental results is rather good (Fig. 13). The impact on the wing is shown in Fig. 14. At same Alpha, the HTP has very little impact on the wing (only visible at the shock position). Looking at the effect at same lift, the presence of the HTP leads to significant differences: to compensate the HTP negative lift, the wing produces greater suction levels.

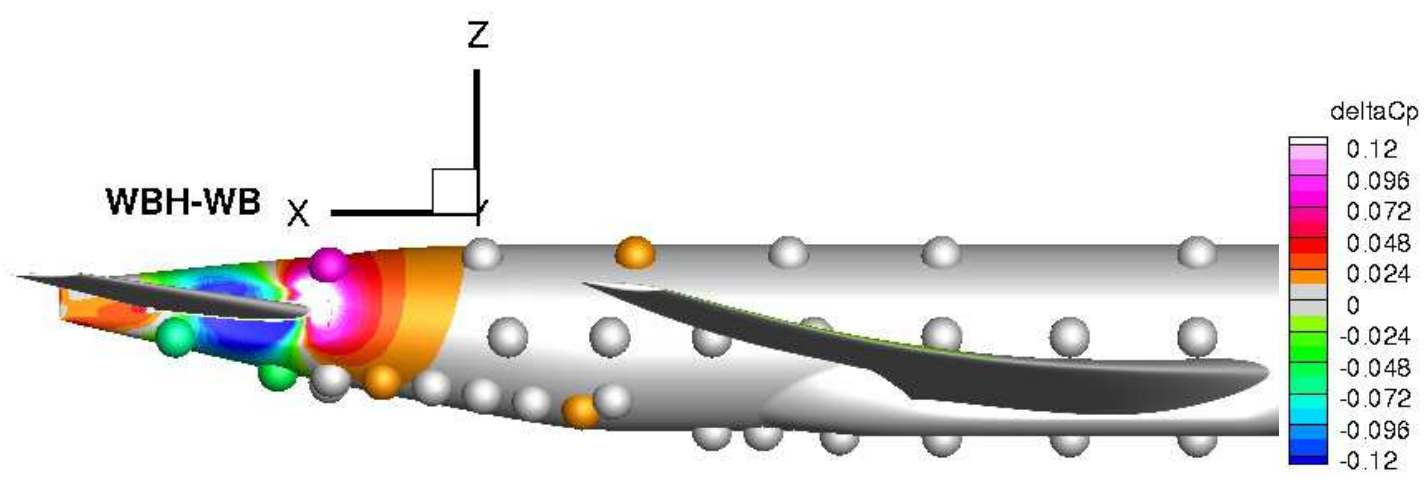

Fig. 13 Pressure coefficient disturbance on model skin (WBH minus WB) at $M a=0.85$ and $C L=0.5$. 

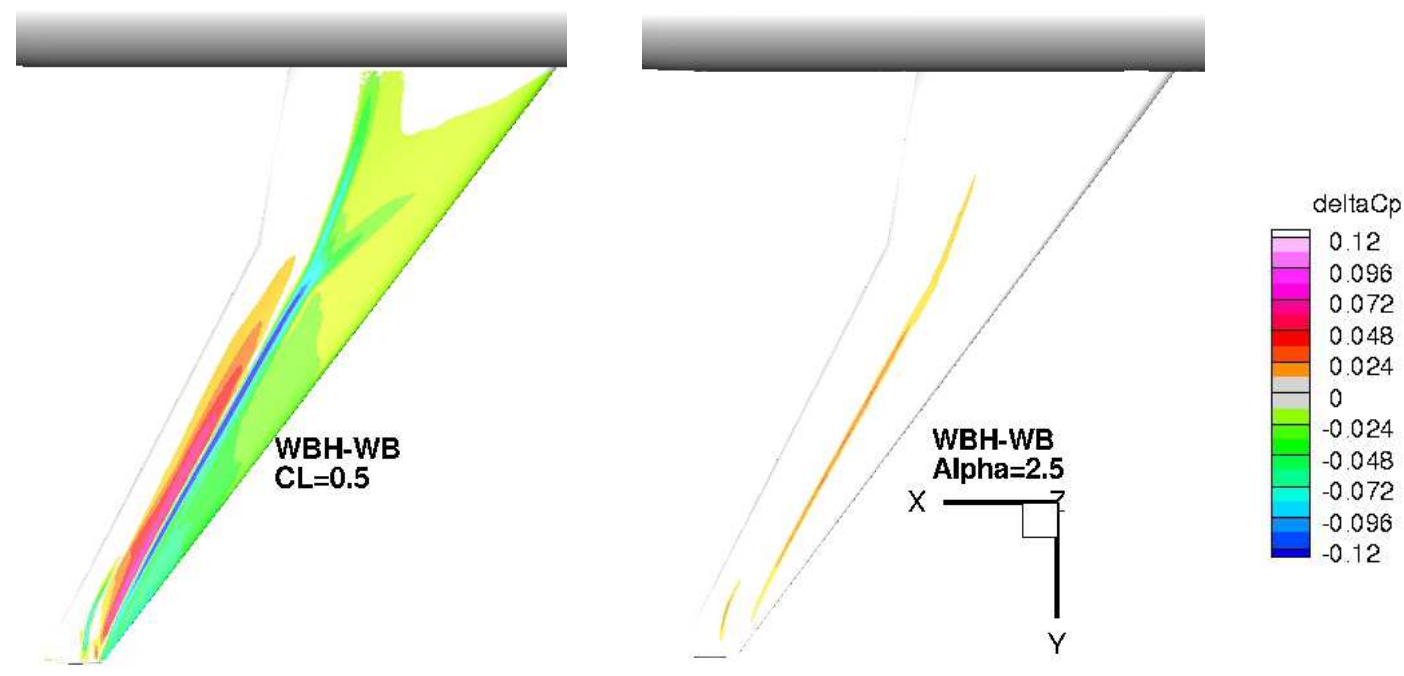

Fig. 14 Pressure coefficient disturbance on the wing (WBH minus WB) at $M a=0.85$.

\section{B. VTP effect without HTP}

The VTP effect without HTP is then investigated. Fig. 15 presents the experimental effects at $M a=0.85$. This figure shows that VTP effect without HTP has mainly a drag component due to the VTP drag itself, which is about 10 counts, as calculated in [9].

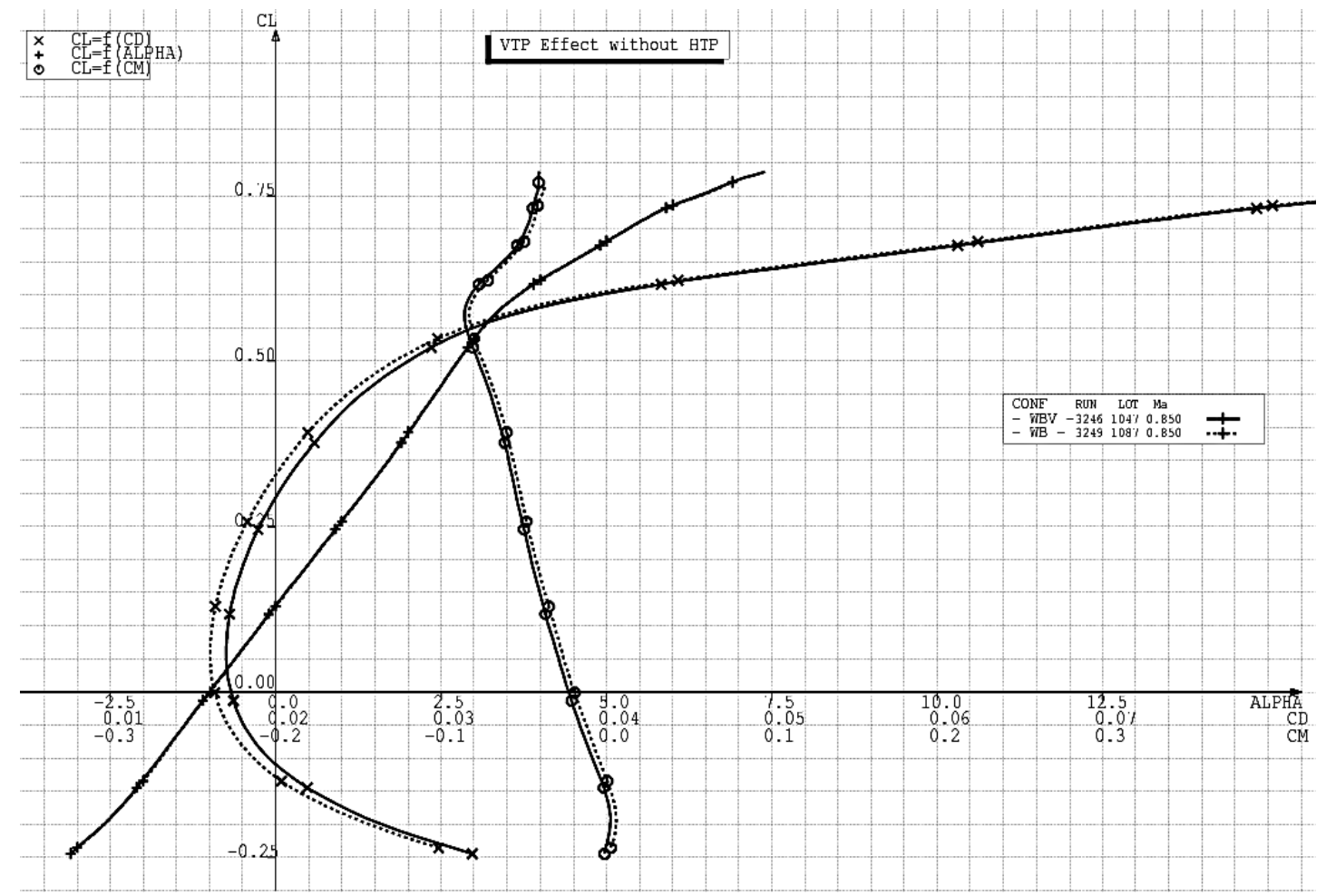

Fig. 15 Experimental VTP effect without HTP at $M a=0.85$ and $C L=0.5$. 


\section{VTP effect with HTP}

The VTP effect with HTP is finally investigated. Fig. 16 shows the experimental effects at $M a=0.85$. The addition of vertical tailplane to the WBH configuration increases the drag (for $C L<0.55$ ), increases the lift and lowers the pitching moment.

The effect is presented in increments in Fig. 17 for Mach number values of 0.7, 0.85 and 0.87. At $M a=0.85$, the effects are globally constant with lift, up to $C L=0.5$. The pitching moment effect increases with Mach number. For $C L=0.5$ and $M a=0.85$, the tail effect is about $-0.1^{\circ}$ of Alpha, 10 counts of drag consistently with the former section and -0.05 of pitching moment. The agreement between numerical and experimental effects is satisfactory. The VTP effect on the near-field drag coefficients is shown in Fig. 18. The impact is mainly due to the friction drag up to $C L$ $=0.5$ and is consistent with an increase of $8 \%$ of the wetted surface area (between WBVH and WBH configurations). The negative lift level generated by the HTP is weaker due to the presence of the VTP leading to a higher global lift and a lower pitching moment.

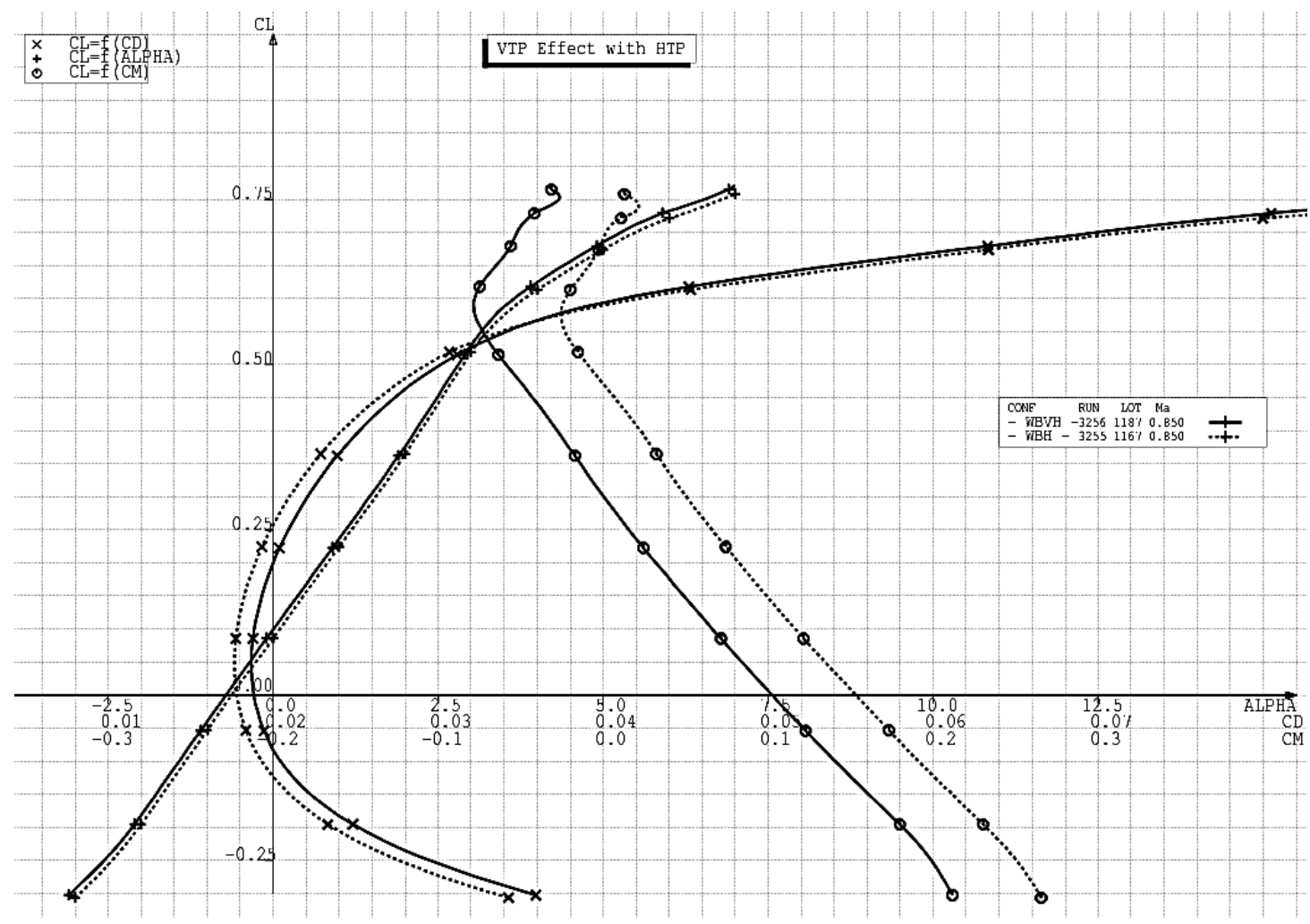

Fig. 16 Experimental VTP effect with HTP at $M a=0.85$ and $C L=0.5$. 

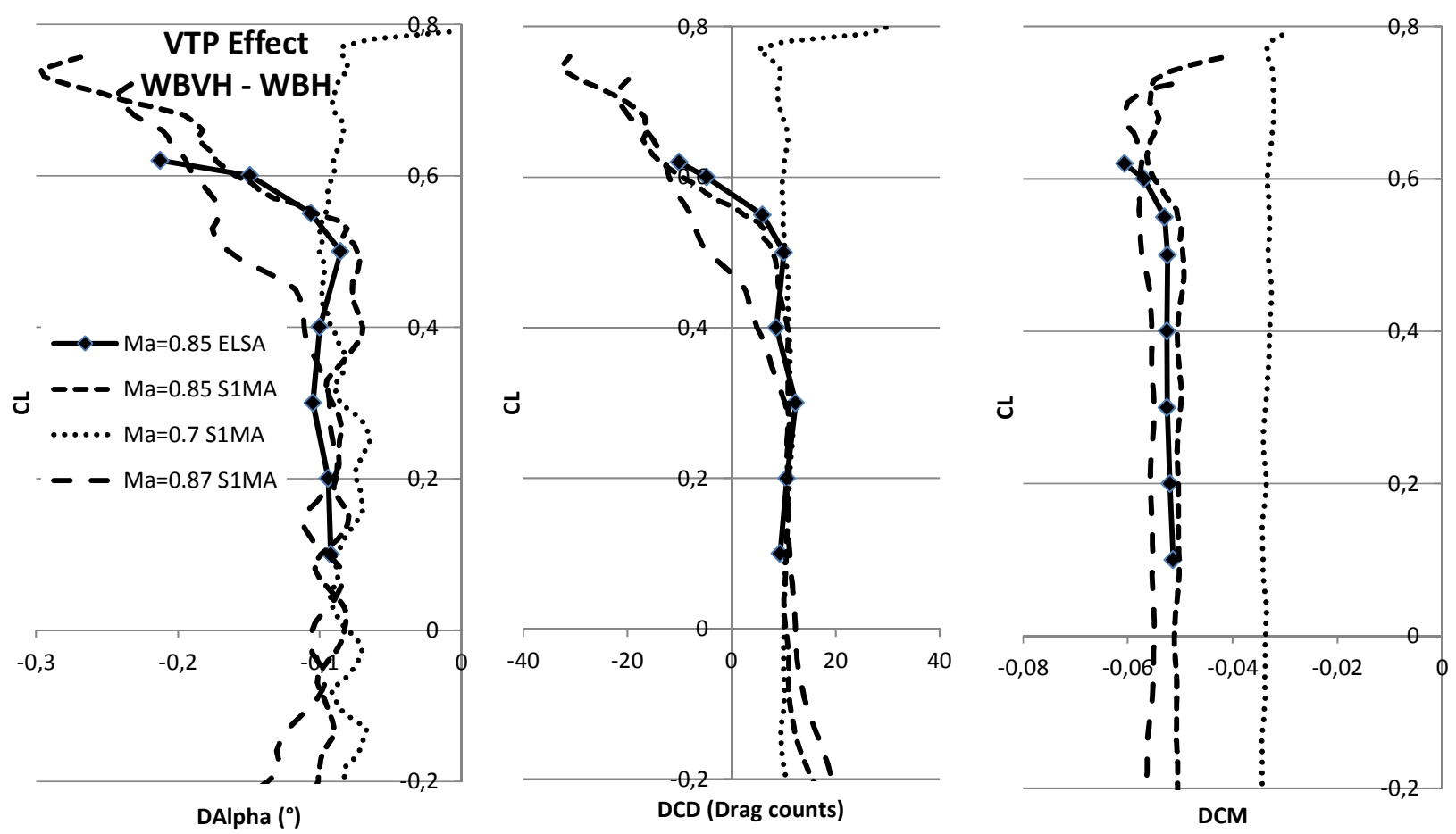

Fig. 17 Numerical and experimental VTP effect (WBVH minus WBH).

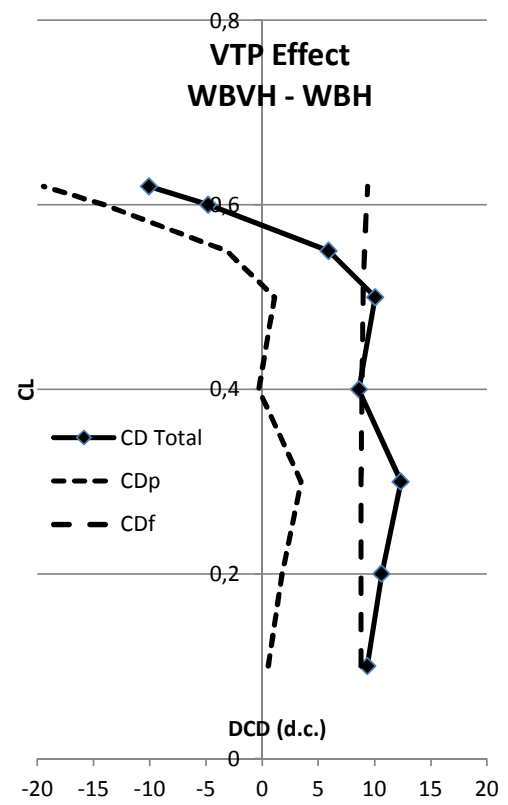

Fig. 18 Numerical VTP effect on near field drag at $M a=0.85$. 
Flow distortion between WBVH and WBH configurations can be observed in Fig. 19 that shows pressure coefficient delta on the model skin at $C L=0.5$. Experimental results are added to numerical results. The larger disturbances are logically observed on the fuselage close to the VTP but they extend to the HTP area. The effective local incidence on the rear part of the model is increased by the VTP, which is visible on HTP lifting surface.

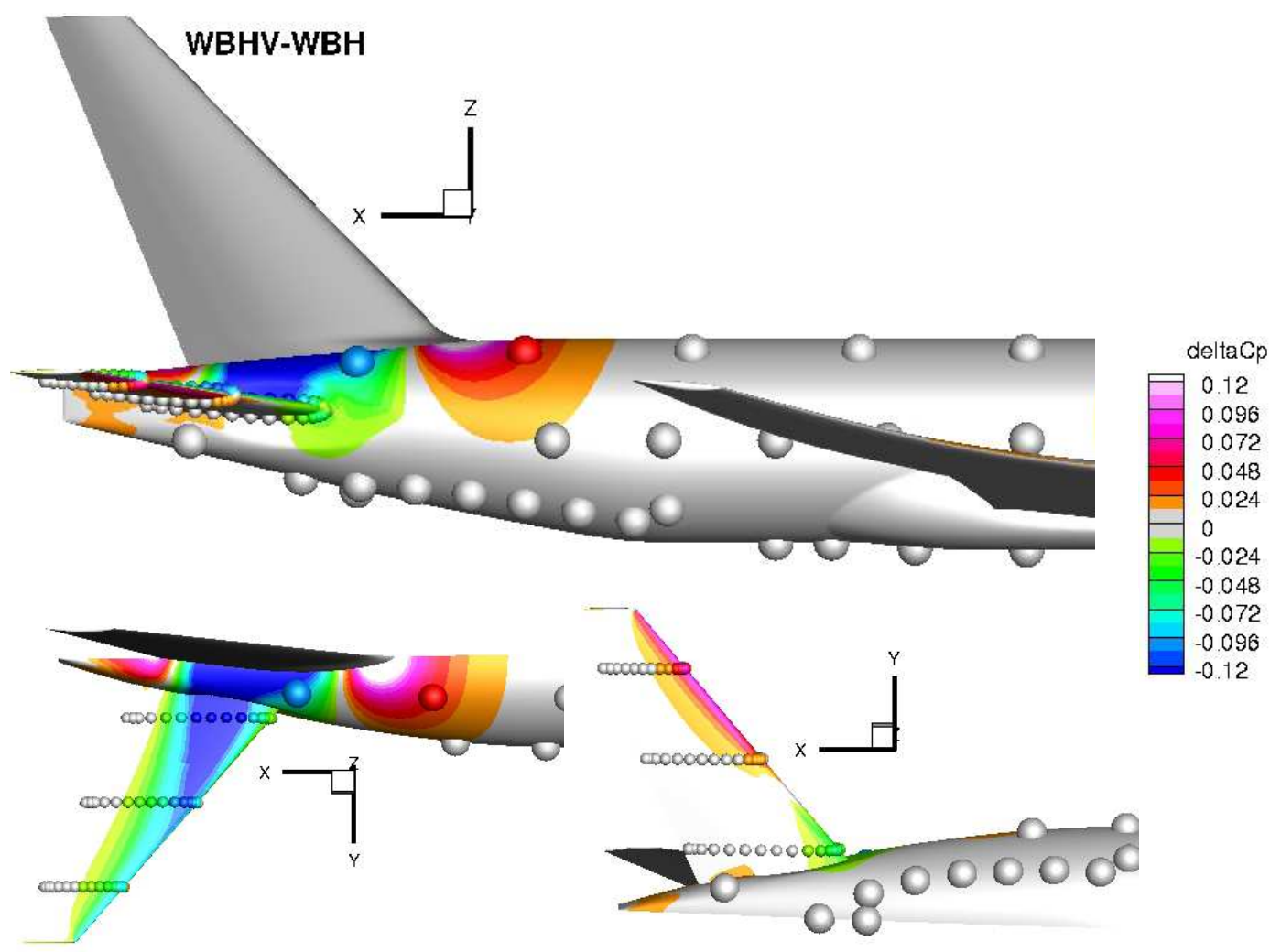

Fig. 19 Pressure coefficient distortion on model skin (WBVH minus WBH) at $M a=0.85$ and $C L=0.5$.

\section{Data repeatability}

When data is obtained in any experimental investigation it is important to make an assessment of data repeatability. "Short term repeatability" is the comparison of polars performed within the same run or within successive runs for a given configuration. Within each series of runs, two polars were obtained at $M a=0.85$. Fig. 20 presents the superposition of all the short term repeats performed at $M a=0.85$ during the test campaign. Lift is presented versus delta angle of attack, drag and pitching moment. The deltas represent the difference between the coefficient value measured and the average value of the coefficient at fixed $C L$. This short term repeatability is good (repeatability better than $\pm 0.02^{\circ}, \pm 2$ d.c. and $\pm 0.001 \mathrm{CM}$ ) and is in compliance with the expected standards for a full span model campaign performed over a large test domain. 


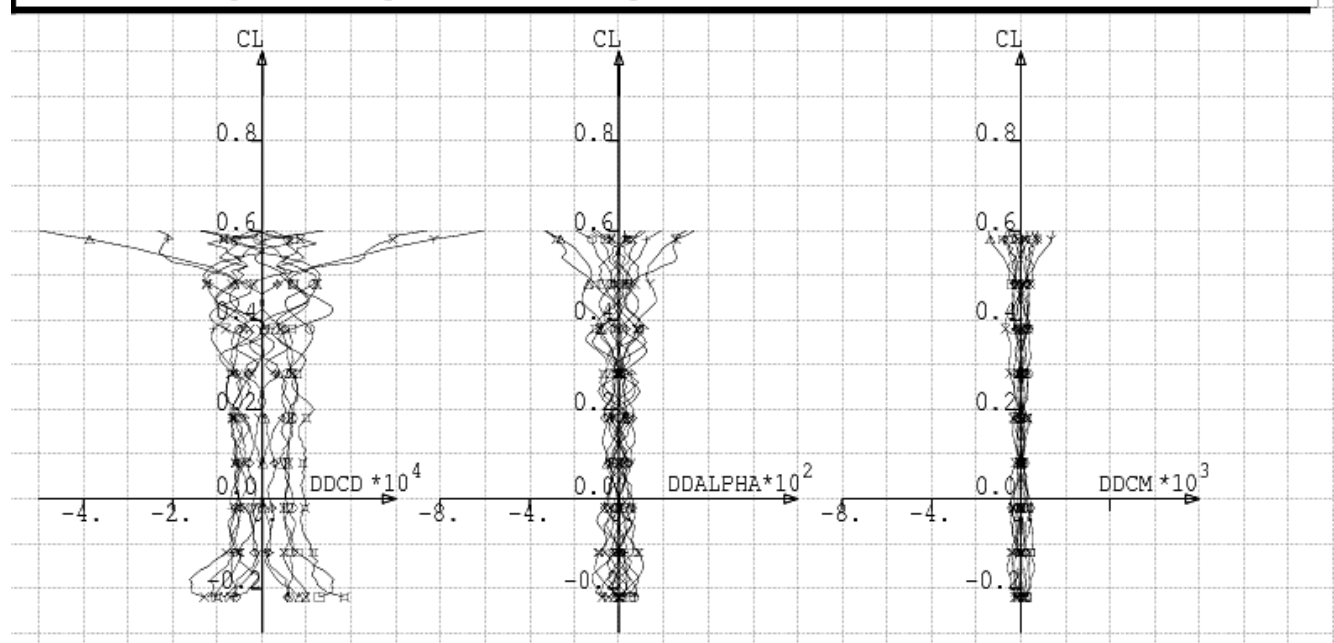

Fig. 20 Short term repeatability at $M a=0.85$.

"Long term repeatability" is the comparison of polars performed during different runs with other configurations tested in-between. Fig. 21 presents the long term repeatability obtained for the WBVH configuration performed at $M a=0.85$. The short term repeatability (in dashed lines) is added to the comparison. The scatters between the four interpolated polars do not exceed $\pm 0.02^{\circ}, \pm 2$ d.c. and $\pm 0.001 C M$, which is the same order of magnitude as with the short term repeatability.

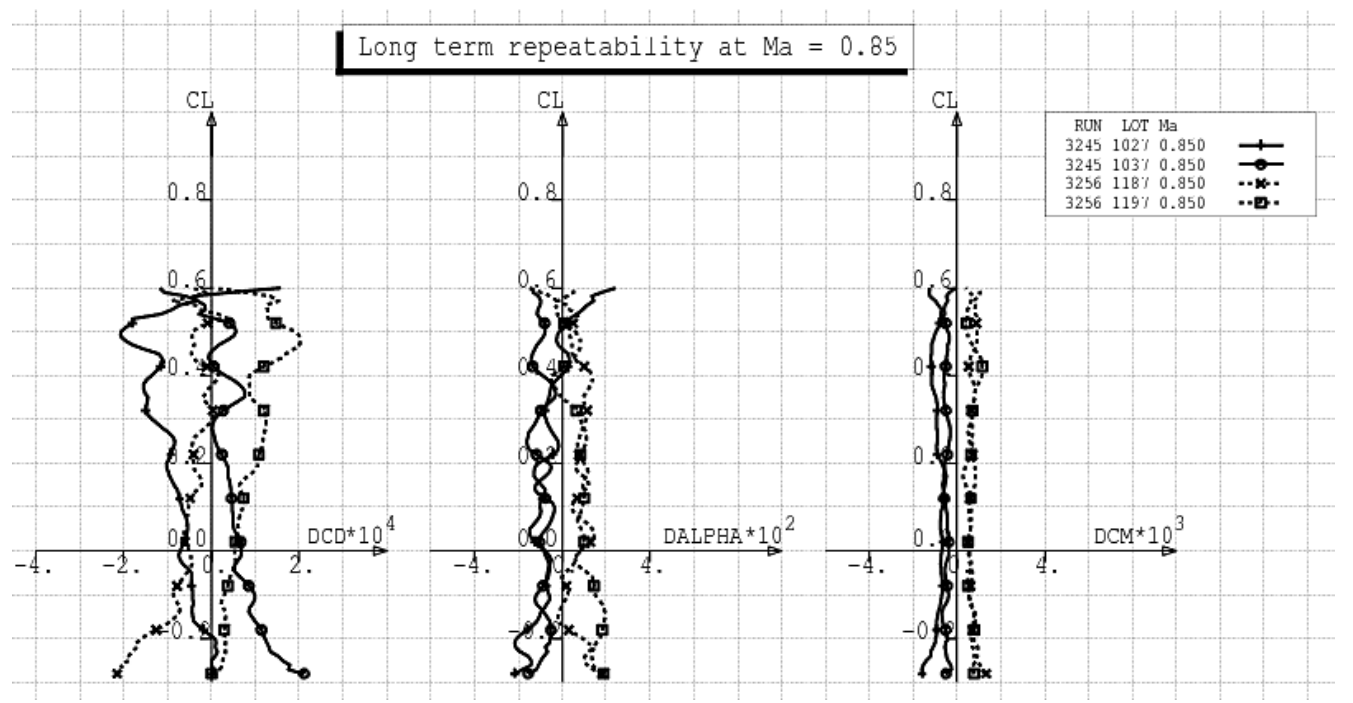

Fig. 21 Long term repeatability for $\mathrm{WBVH}$ at $M a=0.85$. 


\section{Wing-Body junction Flow separation}

The issue of flow separation at the wing trailing-edge/body junction has been frequently addressed in the past Drag Prediction Workshops. It is of academic and industrial interests, for instance the underestimation of a massive corner flow separation can make inaccurate the performance assessment at cruise but can also trigger flow separations at higher angles of attack. It has been numerically studied by many DPW participants; the effects of grid refinement and turbulence model have been highlighted [3,5,9,25]. This section will focus on the flow separation occurring at design point, i.e. for a lift coefficient of 0.5. First, the experimental data will be presented: so far, S1MA test campaign was the only one which has produced visualizations allowing the characteristics of the flow separation at wing-body junction to be determined. Then, complementary numerical analyses will be given, the computation settings will be discussed according to the agreement with test data.

\section{A. Experimental Visualizations}

At the end of this first test campaign, some colored oil flow visualizations were performed. The aim was to identify a corner flow separation at the wing fuselage intersection. The visualizations were performed on the WBVH configuration at $M a=0.85, C L=0.5$ and a Reynolds number of 5 million. The wind-off pictures are shown in Fig. 22. The wing suction side is in blue while the wing pressure side and fuselage are respectively in red and yellow.
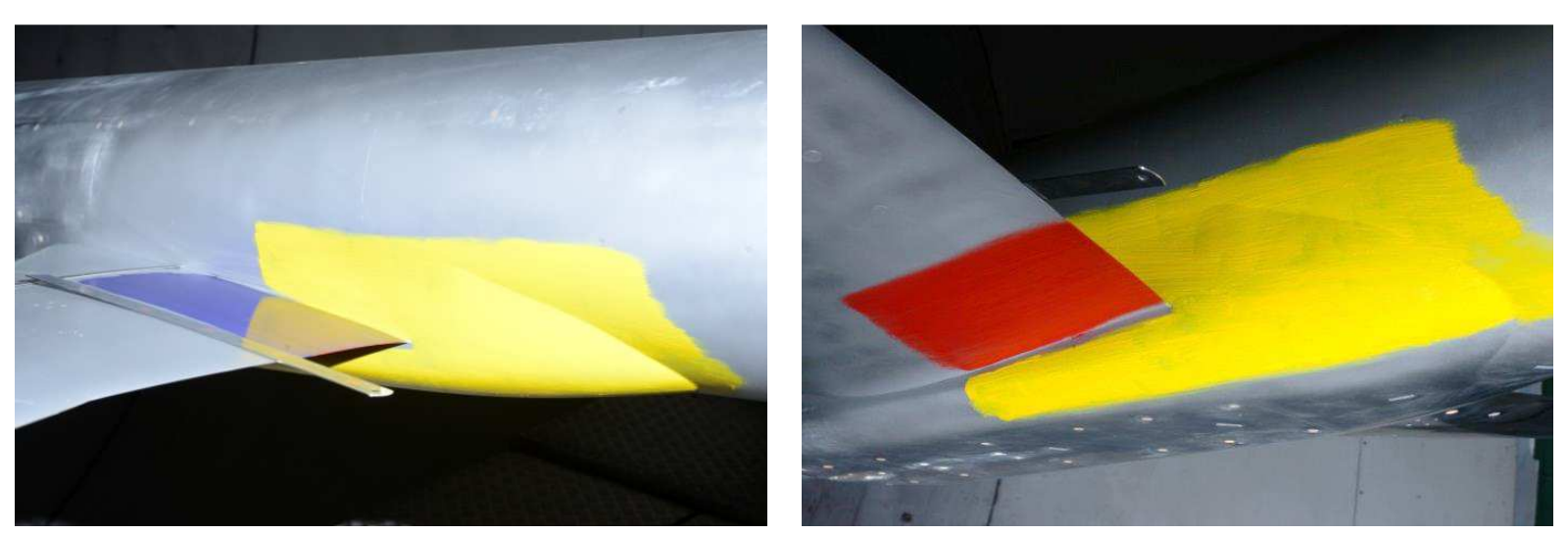

Fig. 22 Wind-off colored oil visualization.

After the run, a flow separation zone was indeed observed at the wing trailing-edge/fuselage intersection as shown in Fig. 23 for the body and in Fig. 24 for the wing suction side (top view). The separation zone on the fuselage near the wing-fuselage junction was not observed by oil flow visualizations in [26] but stream lines on the fuselage were similar and possibly an indicator for that separation. 

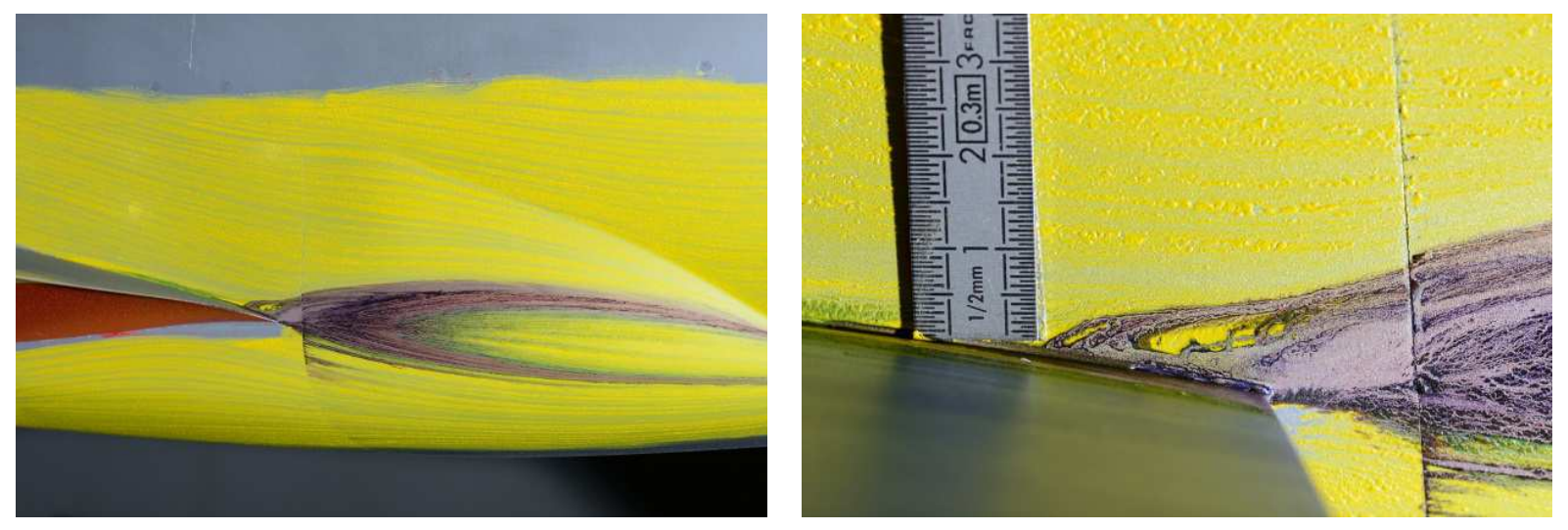

Fig. 23 Fuselage flow separation.

The wing flow separation will be studied with more attention; it can be observed in Fig. 24 that its width at trailing edge is between 1.25 and $1.55 \mathrm{~cm}$. At scale 1/1 (real aircraft dimensions), it represents a width between 21 and $26 \mathrm{~cm}$ (i.e. $0.71-0.88 \%$ of semispan). The length is more difficult to measure; there is more uncertainty about the position at where the flow separation really starts. The values at scale 1/1 that will be considered are: 120-205 $\mathrm{cm}$ (about 10 to $20 \%$ of root chord). In the next LRM campaigns at $\mathrm{S} 1$, additional visualizations at higher $C L$ will be performed (for angles of attack around 3.0 to $4.0^{\circ}$ ).

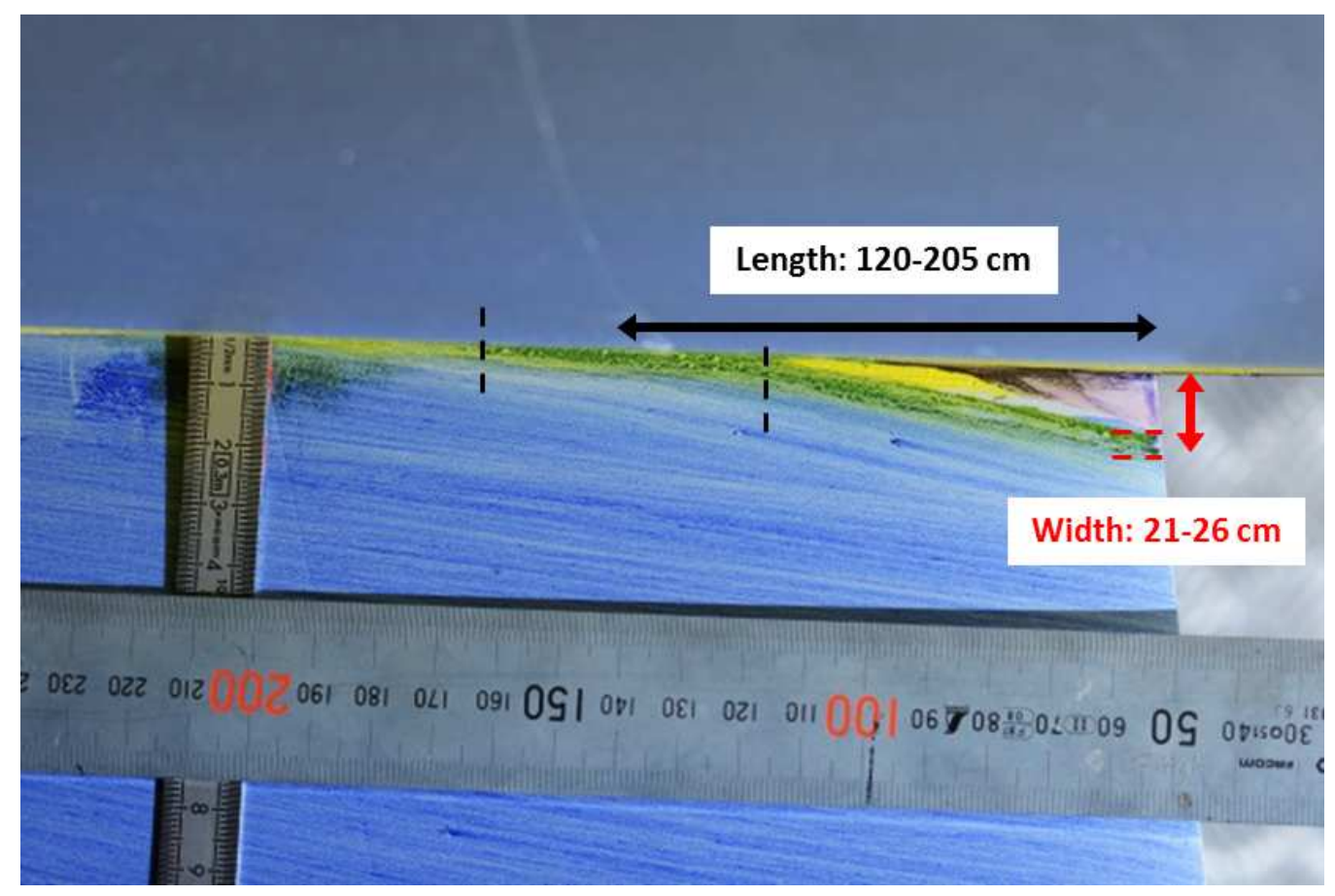

Fig. 24 Side-of-body wing flow separation (top view). 


\section{B. Numerical Analyses}

In this sub-section, an analysis of different CFD results focusing on the side-of-body flow separation prediction is proposed. As mentioned previously, this delicate issue has been widely discussed. To be consistent with the former numerical studies on the subject, this is the WB configuration which will be used (whereas the flow visualizations of $\mathrm{S} 1$ have been done on the WBVH geometry). It has been verified with adequate computations that the flow separation that appears at the wing-body junction of the WB configuration at cruise lift can be considered as identical to the one of the WBVH configuration.

The focus will be here on the flow separation occurring on the wing; the one on the fuselage being noticeably smaller. The main characteristics that will be considered are the flow separation width and length (respectively $\mathrm{BL}_{\text {bub }}$ and $\mathrm{FS}_{\text {bub }}$ according to the DPW-5 Nomenclature [25]). The computations of DPW-5 and DPW-6 geometries with different grids and models will be reviewed. Even if the DPW-6 wing has been modified (see section III), there is no shape difference in the flow separation area. Only negligible effects of the wing geometry modification are expected on the flow separation features at design point. It has been verified with the available DPW-6 Overset mesh of the unmodified geometry (i.e. with the original DPW-5 wing shape).

In the framework of DPW-5, the numerical results of many participants have been analyzed [25]. The wing flow separation prediction exhibited a strong sensitivity to the grid refinement. For the finest grids, the flow separation width average was around $30 \mathrm{~cm}$ but with a quite large dispersion (about $+/-7 \mathrm{~cm}$ ). The length average was about 80 $\mathrm{cm}$ but with a greater dispersion $(+/-40 \mathrm{~cm})$. Still in the context of DPW-5 studies, some ONERA results [5] obtained with the Common Multi-Block grid family $\mathrm{L}_{\mathrm{i}}$ (point-matched structured O-type grids) are now presented. The flow separation obtained with elsA and the standard Spalart-Allmaras (SA) model on the fine grid L4 (17 million cells) is shown in Fig. 25 as well as the corresponding grid refinement in the area. The equivalent data for the finest grid L6 (138 million) is given in Fig. 26. For the fine grid, the flow separation is about $14 \mathrm{~cm}$ wide and 20$25 \mathrm{~cm}$ long while for the super-fine grid, it is $23 \mathrm{~cm}$ wide and $55-65 \mathrm{~cm}$ long.

As a conclusion, the flow separation features are indeed strongly dependent on the refinement level in this case. For the finest grid, the width that is obtained is in good agreement with the experiments but the length seems significantly underestimated. 


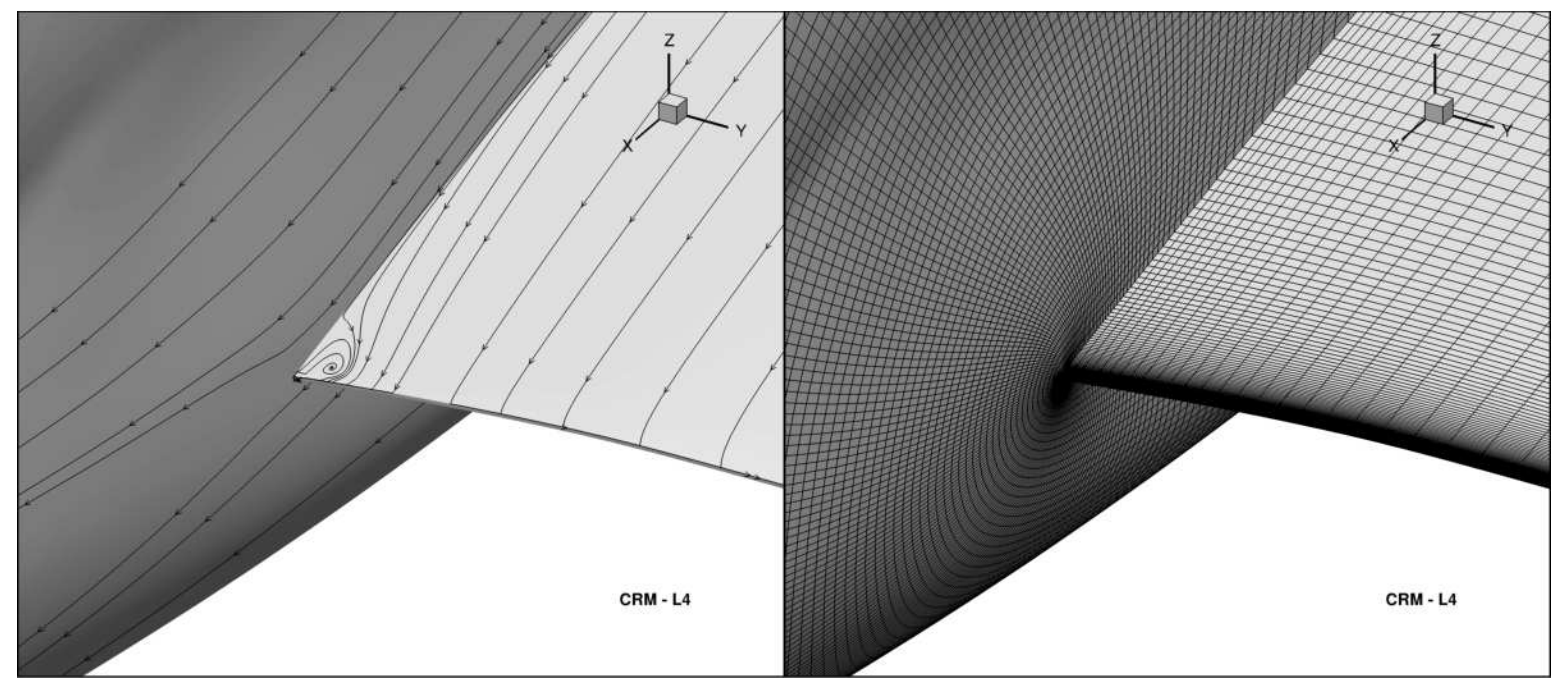

Fig. 25 Side-of-body flow separation; DPW-5 grid L4; SA.

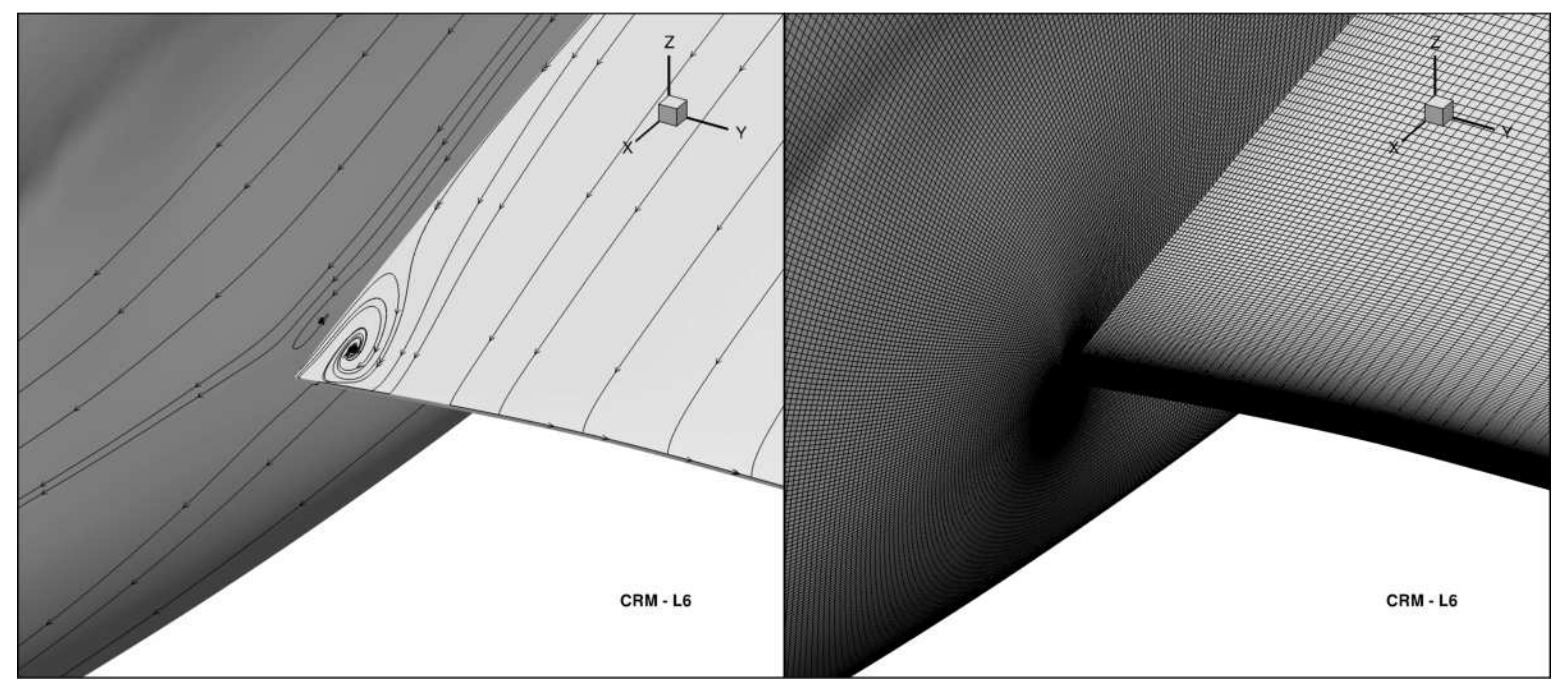

Fig. 26 Side-of-body flow separation; DPW-5 grid L6; SA.

Fig. 27 presents some of the results given in [9]. The geometry is still the one of DPW-5 but the grid is an ONERA Overset mesh named O1. It shows a refinement level equivalent to L4 (17 million cells). Nevertheless, as it can be noticed in Fig. 27, the grid at the wing-body intersection is much more refined in this case, both along the spanwise and normal directions (the grid topology is different). The flow separation which is obtained with elsA and the SA model has a width of about $25 \mathrm{~cm}$ which is almost the same as the one of L6. However, its length is dramatically increased and reaches about $120 \mathrm{~cm}$, which is more in agreement with the experimental values. 


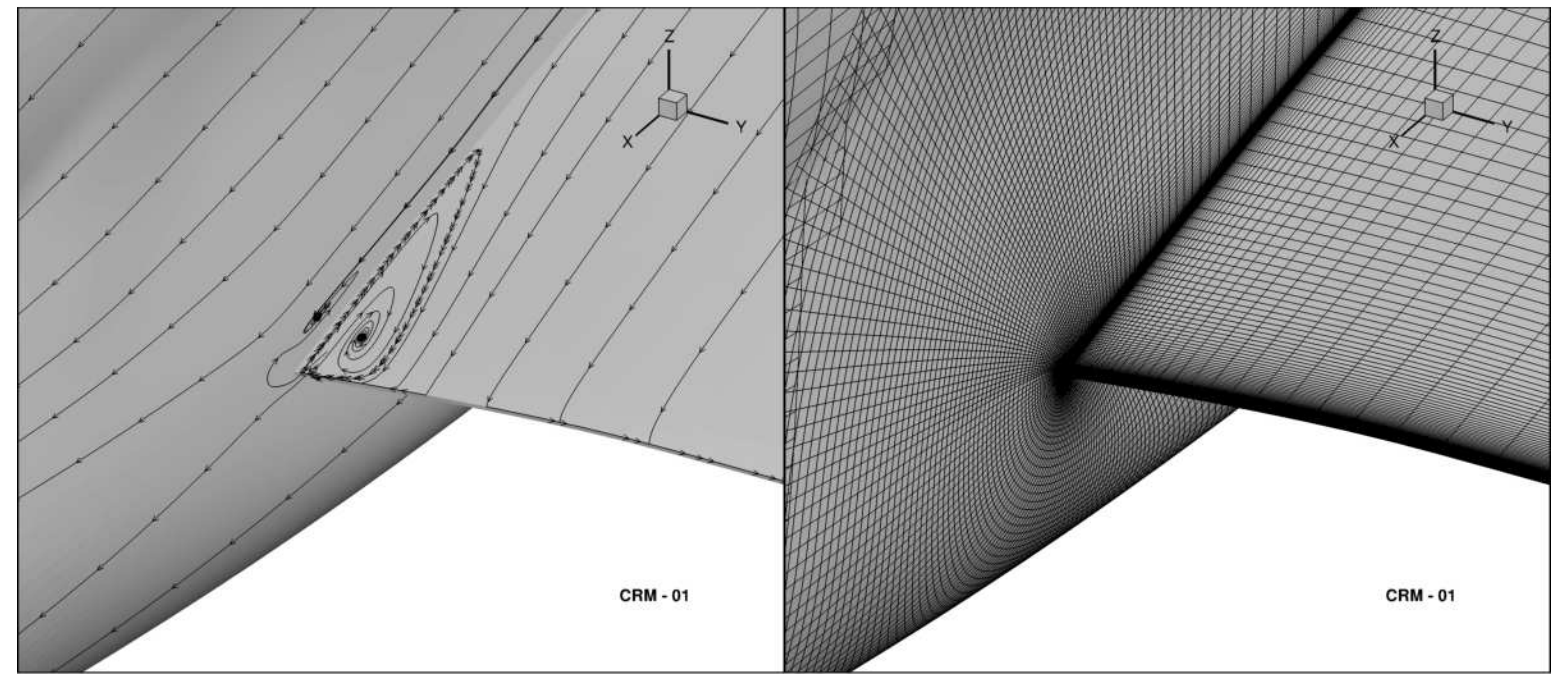

Fig. 27 Side-of-body flow separation; DPW-5 grid O1; SA.

Considering now the DPW-6 geometry (2p75) and Overset grids which have been presented in section III, Fig. 28 and Fig. 29 show respectively the flow separations and refinement levels of the grids WB3 (25 million points) and WB5 (58 million). Contrary to what was observed with the Common grid family used in DPW-5, it seems that in this case the flow separation prediction is not sensitive to the grid refinement. It should be reminded that in the DPW-6 family, the coarse and medium grids are not so coarse. As an illustration, the difference of refinement level between L4 and L6 shown in the previous figures is much more noticeable than between WB3 and WB5. Consequently, there is actually no difference in the flow separation features obtained with the DPW-6 grids from WB1 to WB5. Its width is about $26 \mathrm{~cm}$, which is very close to what was obtained with the ONERA Overset grids and also in good agreement with the experiments. The wing flow separation length that is obtained here is between 120 and $140 \mathrm{~cm}$, which is in the range shown by the S1MA visualization. In the wing trailing-edge/body junction area, DPW-6 grids have characteristics of $\mathrm{L}_{\mathrm{i}}$ and $\mathrm{O}_{\mathrm{i}}$ families. They have the same topology as the $\mathrm{L}_{\mathrm{i}}$ grids at intersection (Navier-Stokes refinement along the corner bisector) but as the $\mathrm{O}_{\mathrm{i}}$ grids, they are Overset meshes. Another potentially important aspect is the stretching of the cells in the chord direction towards the wing leading edge that becomes rapidly strong in the $\mathrm{WB}_{\mathrm{i}}$ grids. 


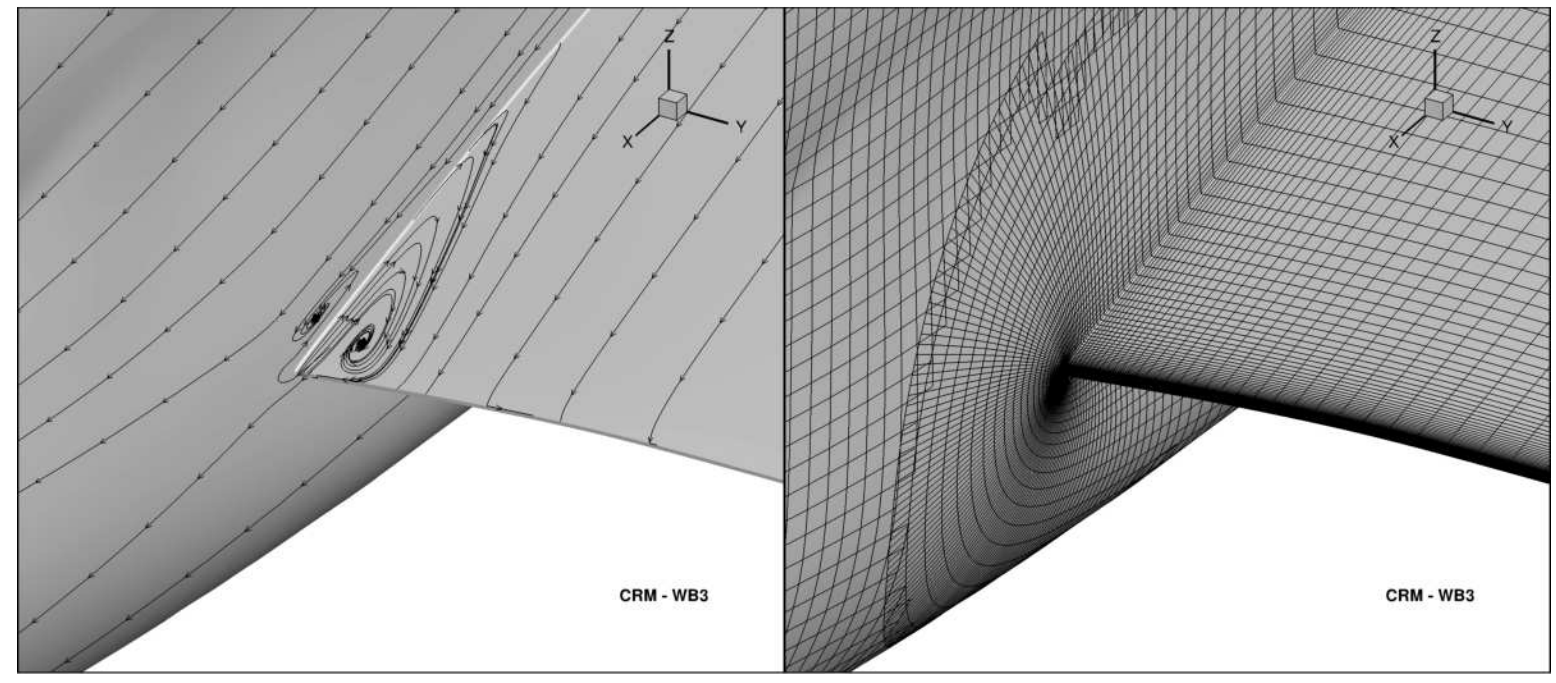

Fig. 28 Side-of-body flow separation; DPW-6 grid WB3; SA.

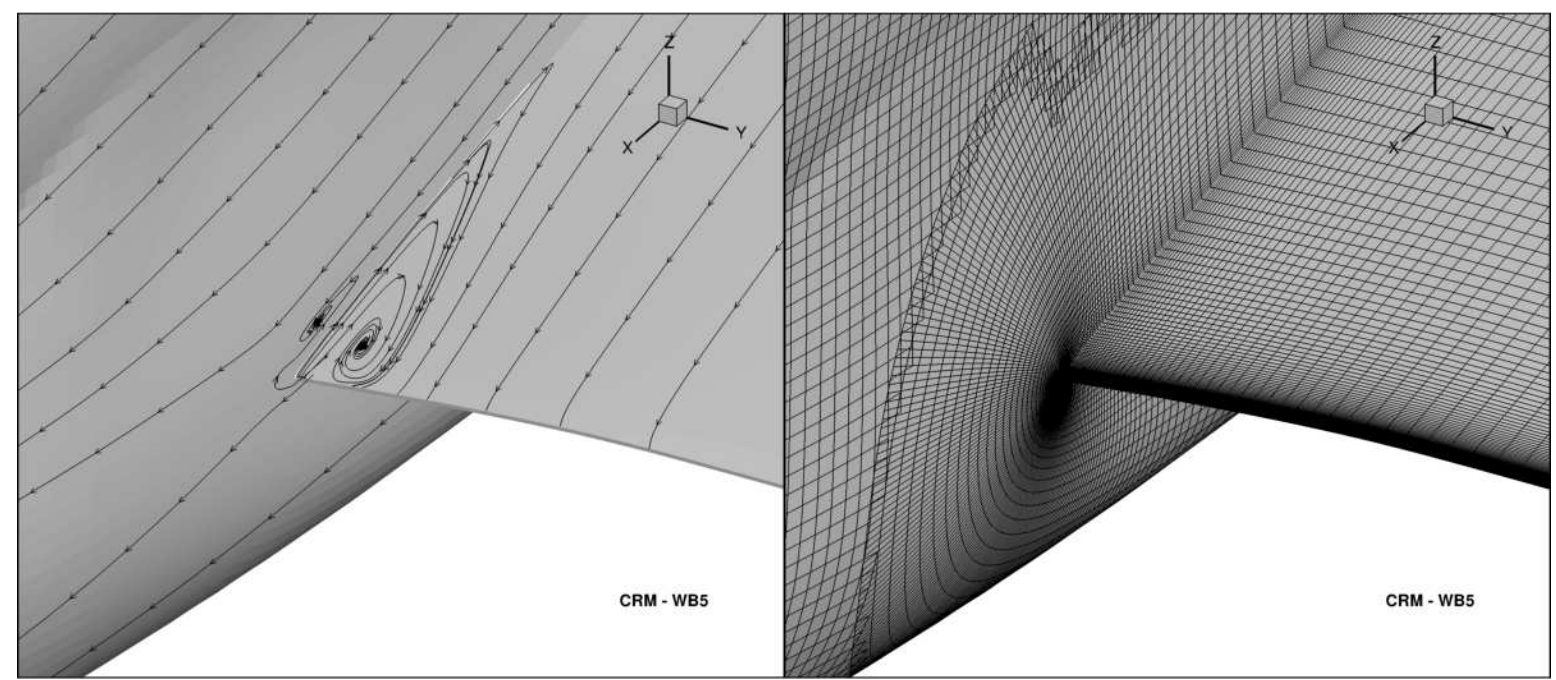

Fig. 29 Side-of-body flow separation; DPW-6 grid WB5; SA.

Beyond the delicate issue of the grid effects, another aspect is the turbulence model. Some additional computations have been performed with the k $\omega$-SST model of Menter presented in section III. The grids O1 and WB3 presented above have been used. In Fig. 30, it can be observed that the flow separations obtained with the k $\omega$ SST model are similar to the ones of SA. However, they are smaller and the eye position is closer to the trailingedge. For the grid O1, the flow separation is $24 \mathrm{~cm}$ wide and about $100 \mathrm{~cm}$ long (versus $25 / 120$ with SA). For the DPW-6 grid WB3, the width is also $24 \mathrm{~cm}$ but the length is limited to $90 \mathrm{~cm}$ (versus 26 / 120-140 with SA). The flow separation length given by k $\omega$-SST might be too limited compared to the wind tunnel data. 


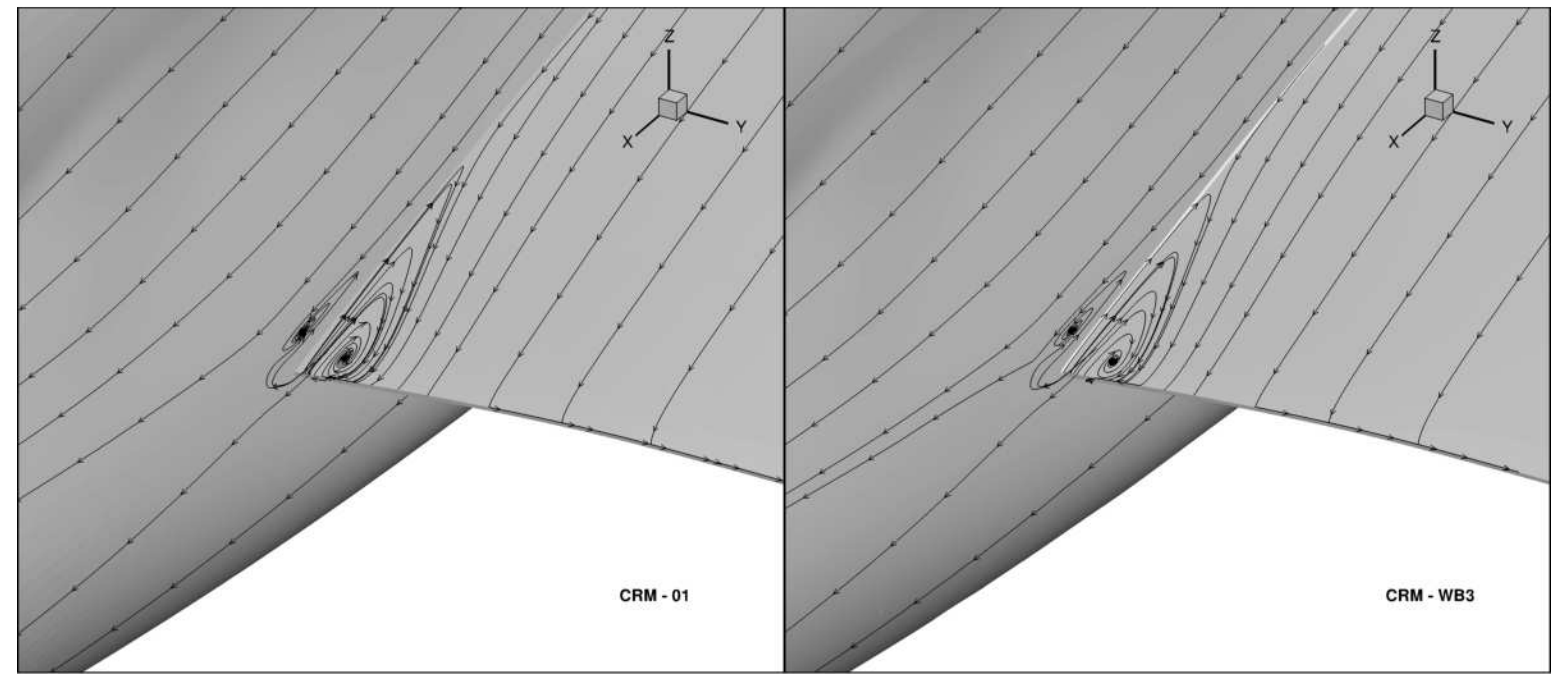

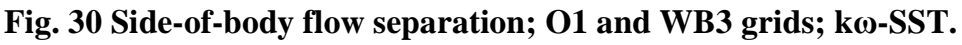

As the Quadratic Constitutive Relation introduced in section III can be beneficial for the performance prediction at lift coefficients greater than the ones of cruise conditions [3], its impact on the side-of-body flow separation at design point has been investigated. In Fig. 31, the results obtained with the SA-QCR2000 implemented in elsA are given. The grids L6 and WB3 have been considered. For the point-matched DPW-5 grid L6 which exhibits the greatest number of cells, the corner flow separation is barely visible. For the Overset grid WB3, the very limited flow separation that is obtained shows a $10 \mathrm{~cm}$ width and a $50 \mathrm{~cm}$ length (versus $26 / 120-140$ with standard SA). The effect of QCR with the k $\omega$-SST model is very similar.

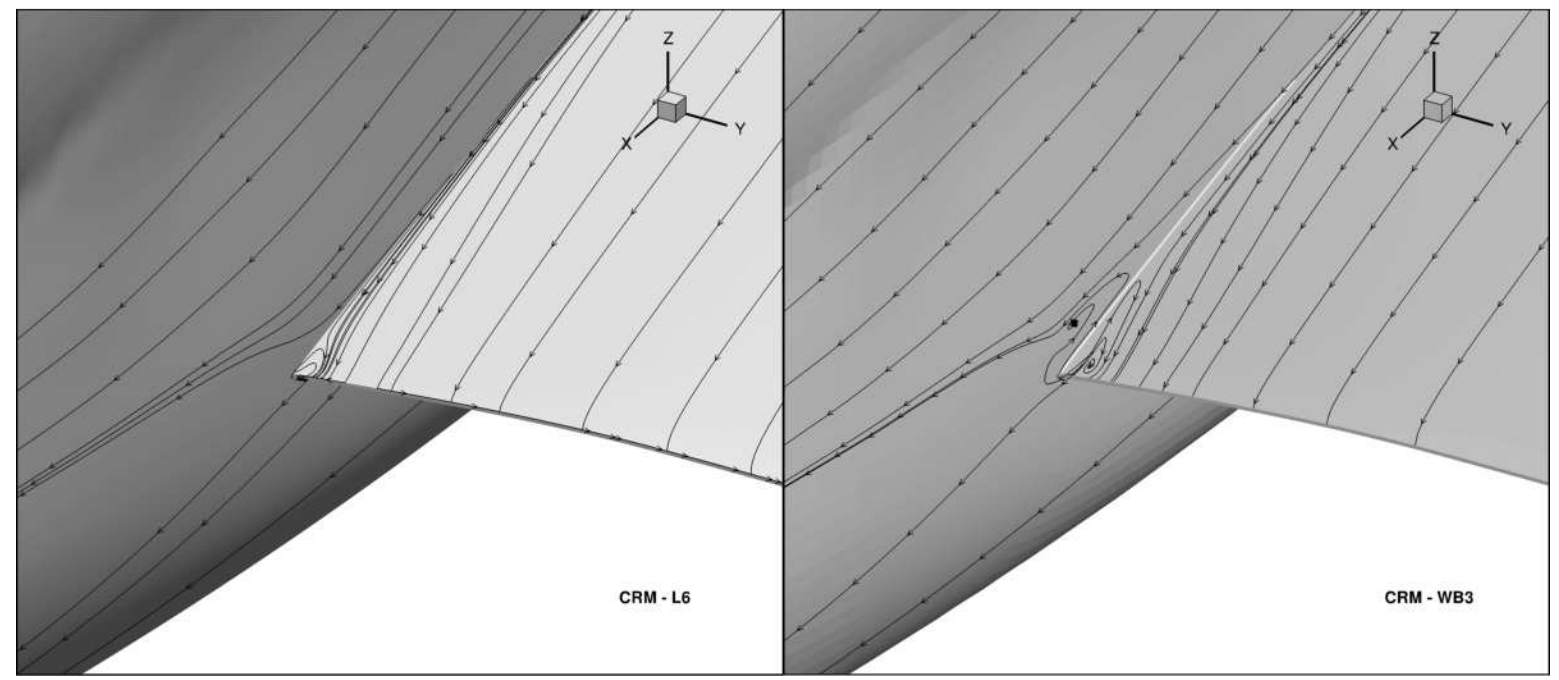

Fig. 31 Side-of-body flow separation; L6 and WB3 grids; SA-QCR2000. 
As a conclusion on this topic of corner flow separation prediction, it should be highlighted that the CFD results are indeed very sensitive to the grid features. Furthermore, it seems that it is not only the refinement level in itself that is the predominant parameter. The grid topology and the cell characteristics (stretching, aspect ratio, skewness) clearly play a significant role.

For the most part of the computations presented above, the side-of-body flow separation width seems to be in good agreement with the wind tunnel data. This is not true anymore if the QCR is activated. On the other hand, the length of the wing flow separation is more difficult to quantify both for numerical and experimental approaches. In CFD, it seems to be a very sensitive quantity.

The Overset grids proposed in the framework of DPW-6 associated with the standard Spalart-Allmaras model produce flow separation predictions that are in the range of experimental values. In this case, it seems that the k $\omega$ SST model generates flow separations that are similar to the ones of SA but noticeably smaller and with lengths possibly too limited compared to the experiments. It also seems that at design point the QCR leads to flow separations that are probably too small considering the wind tunnel visualization. However, it has been shown in the recent DPW that the use of QCR at angles of attack greater than 3.0-3.5 prevents the development of non-physical side-of-body flow separations.

Obviously, it should be specified that these analyses, even if they have been based on a certain number of computations, are not the conclusions of an exhaustive study. And in transition to the next section, it can be added that given the size of the wing-body junction flow separation existing on the CRM at design point, its accurate prediction has only a minor impact on the global aircraft performance at cruise (typically one to two drag counts).

\section{Experimental/Numerical Comparison}

One major objective of this paper is to compare the data between the NASA wind tunnels, ETW and S1MA, and more specifically the pressure distributions and global coefficients. This is a crucial step for absolute performance prediction and code validation. The comparison is made on the WBH configuration for which results are available in all wind tunnels. The data presented here were obtained at a Mach number of 0.85 and a chord Reynolds number of 5 million. Transition was fixed for all wind tunnel tests (also at $10 \%$ chord).

\section{A. Pressure comparison}

Numerical computations were performed with the same grids and geometries as in section IV, and with the SAQCR2000 model (fully turbulent). The pressure distributions obtained on the WBH configuration at $M a=0.85$ and $C L=0.5$ are shown in Fig. 32. For Ames data, the closest $C L$ was slightly lower. All the data compare relatively well across the entire wing. The first noticeable difference is seen on the outer wing where CFD tends to overpredict the aft-loading. The other difference is seen at $\eta=0.727$. The shock on the wing is located further in the ETW and S1MA data than in the NTF and Ames data.

The numerical simulation for $C L=0.495$ was obtained for an angle of attack of $2.57^{\circ}$ and was calculated with the wing geometry referenced as 2 p50. The impact of using the 2 p 75 geometry on the pressure distribution is insignificant. 


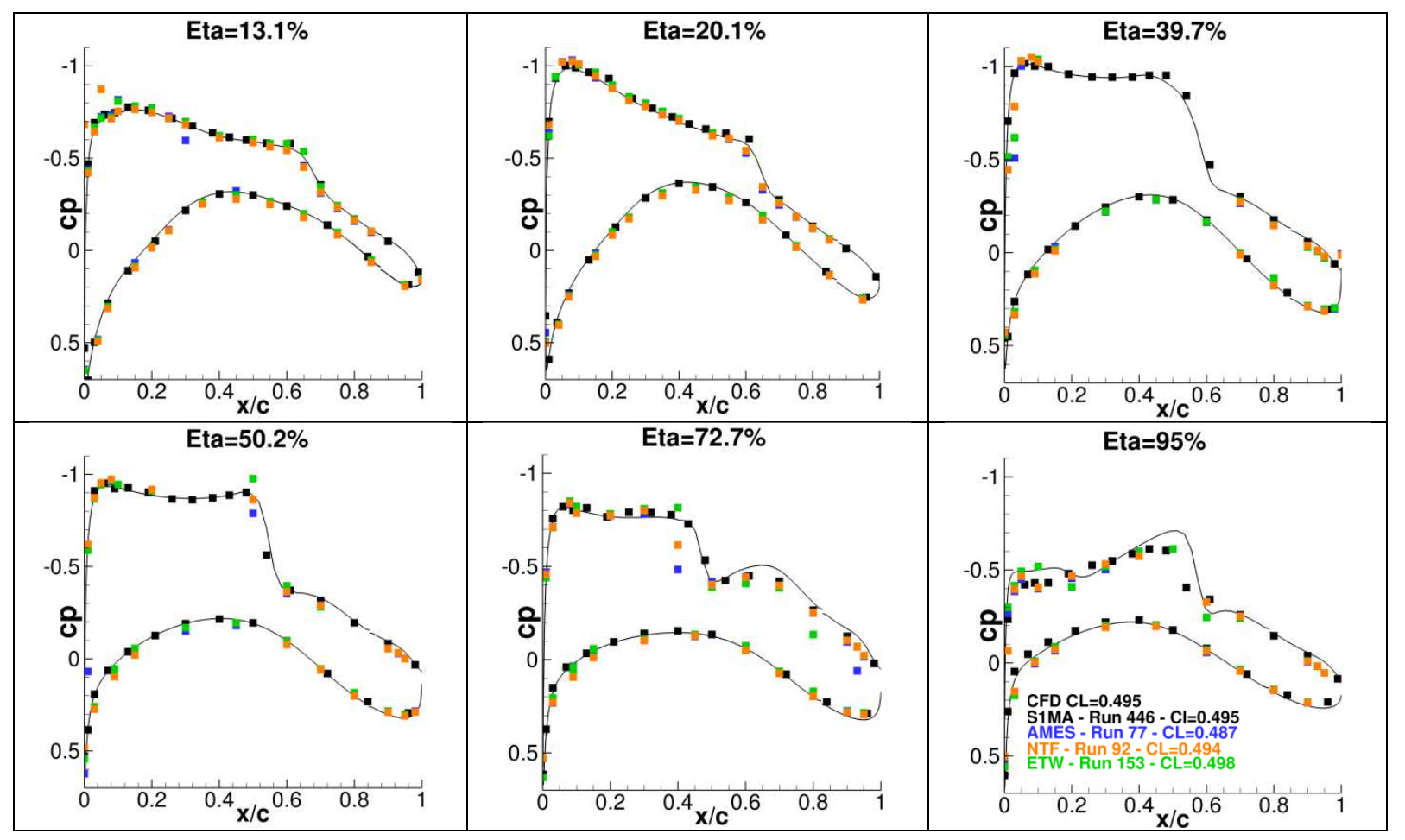

Fig. 32 Pressure distribution for $\mathrm{WBH}$ at $M a=0.85$ and $C L=0.5$.

The pressure distributions obtained on the WBH configuration at $M a=0.85$ and $C L=0.6$ are shown in Fig. 33 . The data shows good consistency in the inner wing. However, from $\eta=0.502$ up to the outer wing, the CFD shock is located further than what is observed in experiments. It has already been noticed when analyzing the DPW-6 outcomes. The agreement remains good on the pressure side and on the suction side before the shock. The shock system at $\eta=0.95$ is not the same between CFD and experiments. However, the agreement between the wind tunnels is rather satisfactory. 


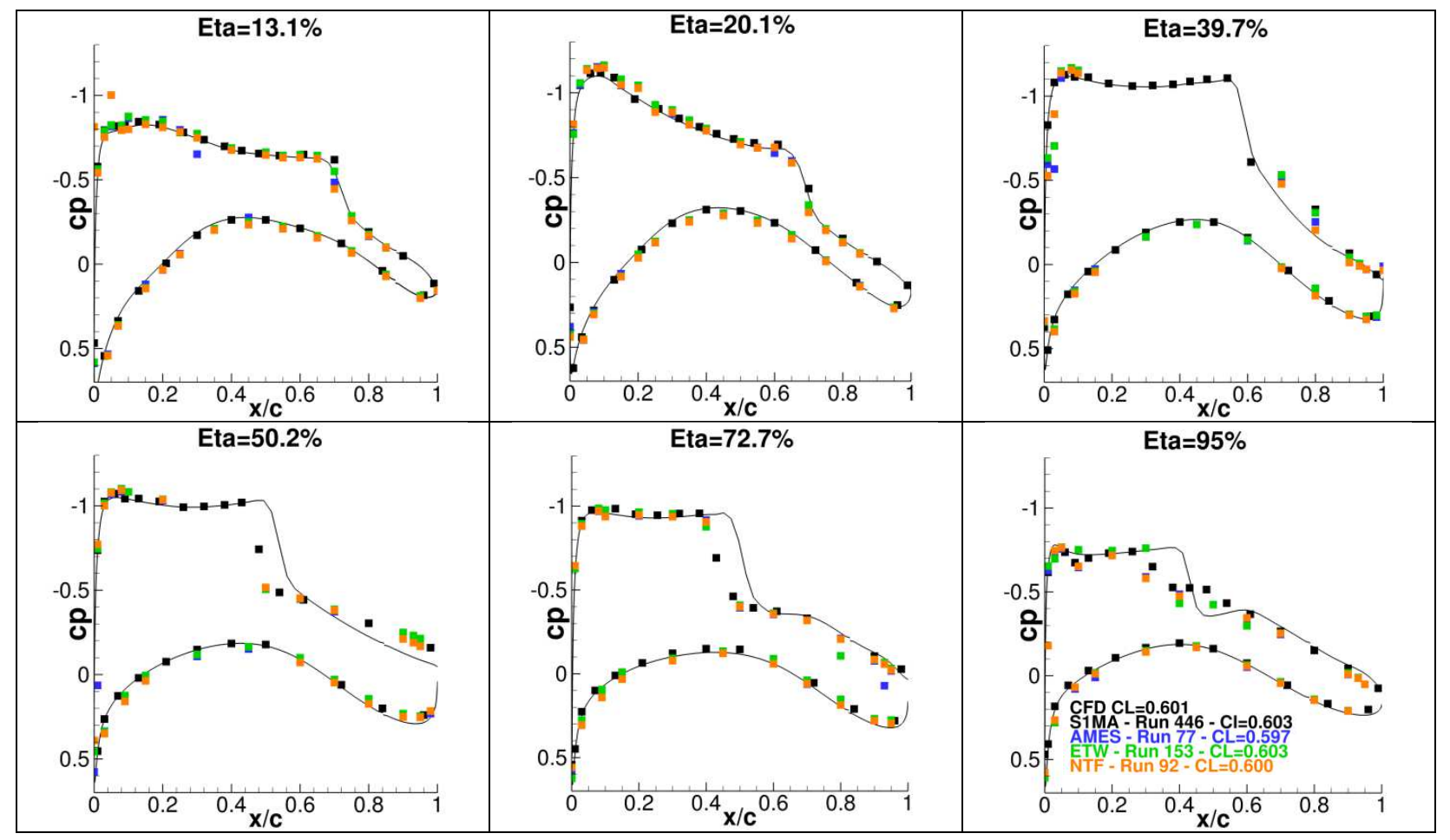

Fig. 33 Pressure distribution for $\mathrm{WBH}$ at $M a=0.85$ and $C L=0.6$.

The numerical simulation for $C L=0.601$ was obtained for an angle of attack of 3.455 degrees and was calculated with the wing geometry referenced as $3 \mathrm{p} 25$. By using the wing geometry referenced as $3 \mathrm{p} 75$, the pressure distribution on the wing is almost unchanged as it can be seen on Fig. 34.
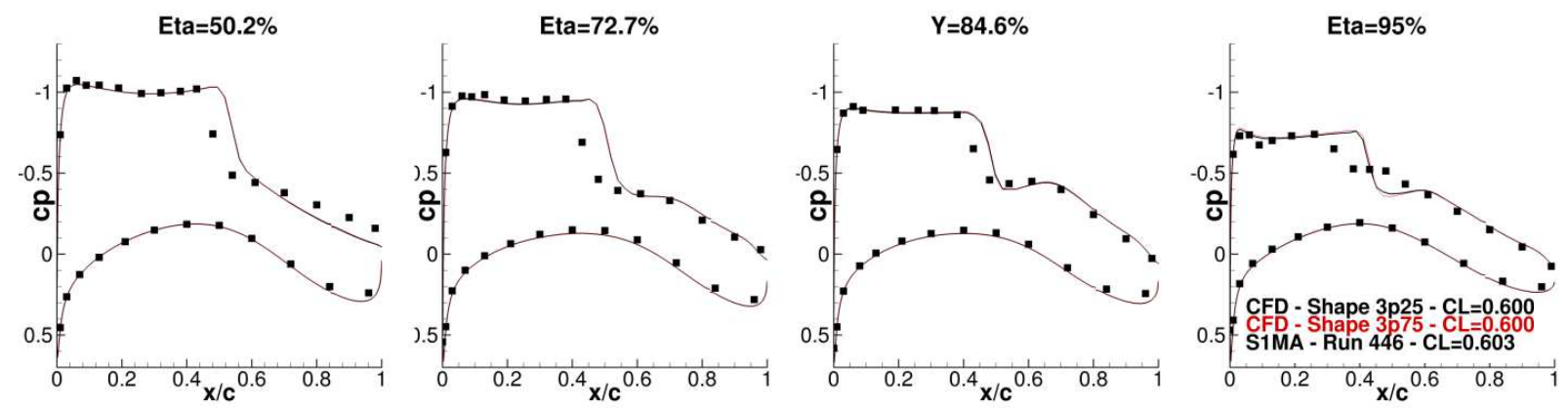

Fig. 34 Pressure distribution for $\mathrm{WBH}$ at $M a=0.85$ and $C L=0.6$, shape effect.

\section{B. Drag and Moment comparison}

The drag polar obtained on the WBH configuration at $M a=0.85$ is first examined. Fig. 35 shows the comparison between the four wind tunnels. For the NASA wind tunnels, wall corrections have been applied [27] but no support corrections. Wall corrections and sting farfield corrections have been applied to the ETW results. Then, it is reminded that S1MA results are corrected for wall and sting interferences. Also, numerical simulations performed by elsA on the grids of section IV are presented: some with the Spalart-Allmaras model and some with the k $\omega$-SST model described in III, both used with the Quadratic Constitutive Relation. 


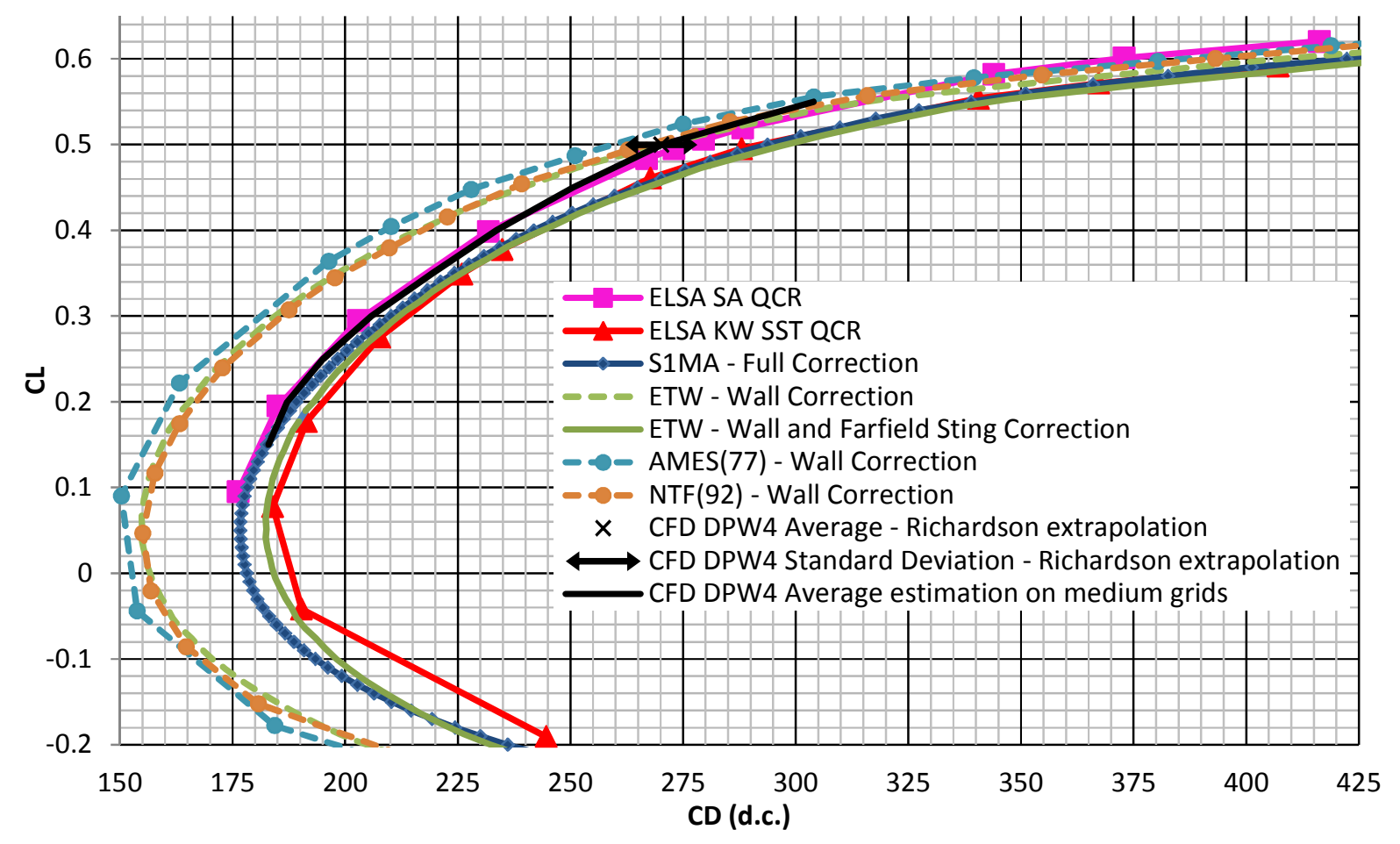

Fig. 35 Drag polar for $\mathrm{WBH}$ at $\mathrm{Ma}=\mathbf{0 . 8 5}$.

First, all NASA and ETW results are similar if not corrected for support effects (dashed line). The same support was used for these three tests. It notably shows that wall corrections are well taken into account.

Then, the comparison between S1MA and ETW is rather good (particularly at high lift coefficients) if ETW data is corrected for wall and farfield support effects, even though two completely different stings are used. This shows that experimental values that are not corrected for support effects have to be considered with caution. Indeed, the ETW farfield support effect is about 25-30 d.c.

Considering now numerical results, Fig. 35 also includes the CFD average obtained from the results of DPW-4 participants at $C L=0.5$ via Richardson extrapolation (table IV of [28]) and an average estimation on medium grids using Fig. 15 of [28] for the other lift levels. The DPW-4 geometry is indeed the WBH configuration; at that time the wing twist was not exactly the one obtained in wind tunnel but this difference produces very little effect on the drag polar as shown in [7]. This CFD average (without outliers) was calculated on the basis of simulations performed with different turbulence models.

It can be observed that the elsA SA-QCR2000 and DPW-4 average curves are in almost perfect agreement over a large $C L$ range. However, what should be noticed here is the fact that the good agreement between these elsASA/DPW-4 computations and the NTF/AMES/ETW data (wall correction only) at design point is purely coincidental.

Indeed, a drag difference of at least 20 d.c. is observed between NTF/AMES/ETW (wall correction) and CFD at $C L=0.1$. And, most important, at design point, a difference of 20-25 counts appears between the elsA-SA/DPW-4 average computations and the fully corrected experimental drag values, which is substantial. 
On the other hand, the CFD results obtained with the k $\omega$-SST turbulence model presented above (pseudoroughness at wall and QCR on) seem to give a better agreement with S1 and ETW (wall and farfield correction), especially for medium and high lift coefficients.

The pitching moment variation with lift is examined in Fig. 36. First, the wind tunnels which are not corrected for support effect show consistent results. Then, the farfield sting corrections from ETW do not include any pitching moment correction so the ETW corrected and not corrected curves are the same. The S1MA support corrections are calculated by CFD and the effect of the support on the pitching moment coefficient is important particularly with the horizontal tail plane. As presented in [15], the NASA support effect calculated by CFD showed an increase of pitching moment $\triangle C M=0.036$ at a lift coefficient around 0.4 . This will significantly reduce the discrepancies between CFD and NTF/AMES/ETW data as depicted by the increment on the pitching moment in Fig. 36. As an additional illustration, the average of DPW-4 computations gave a $C M$ of -0.04 . Contrary to the case of the drag polar above, the fact that there is a wing twist inconsistency between the DPW-4 simulations and the wind tunnel tests do have an impact on the moment value at a given $C L$. Nevertheless, it can be noticed that the S1MA measurements are much closer to the DPW-4 value than NTF/AMES/ETW and also that the DPW-6 computations shown in Fig. 36 exhibit satisfactory agreement with the ONERA wind tunnel data. The pitching moment effect caused by the support is very important and has to be considered when comparisons to numerical results are made.

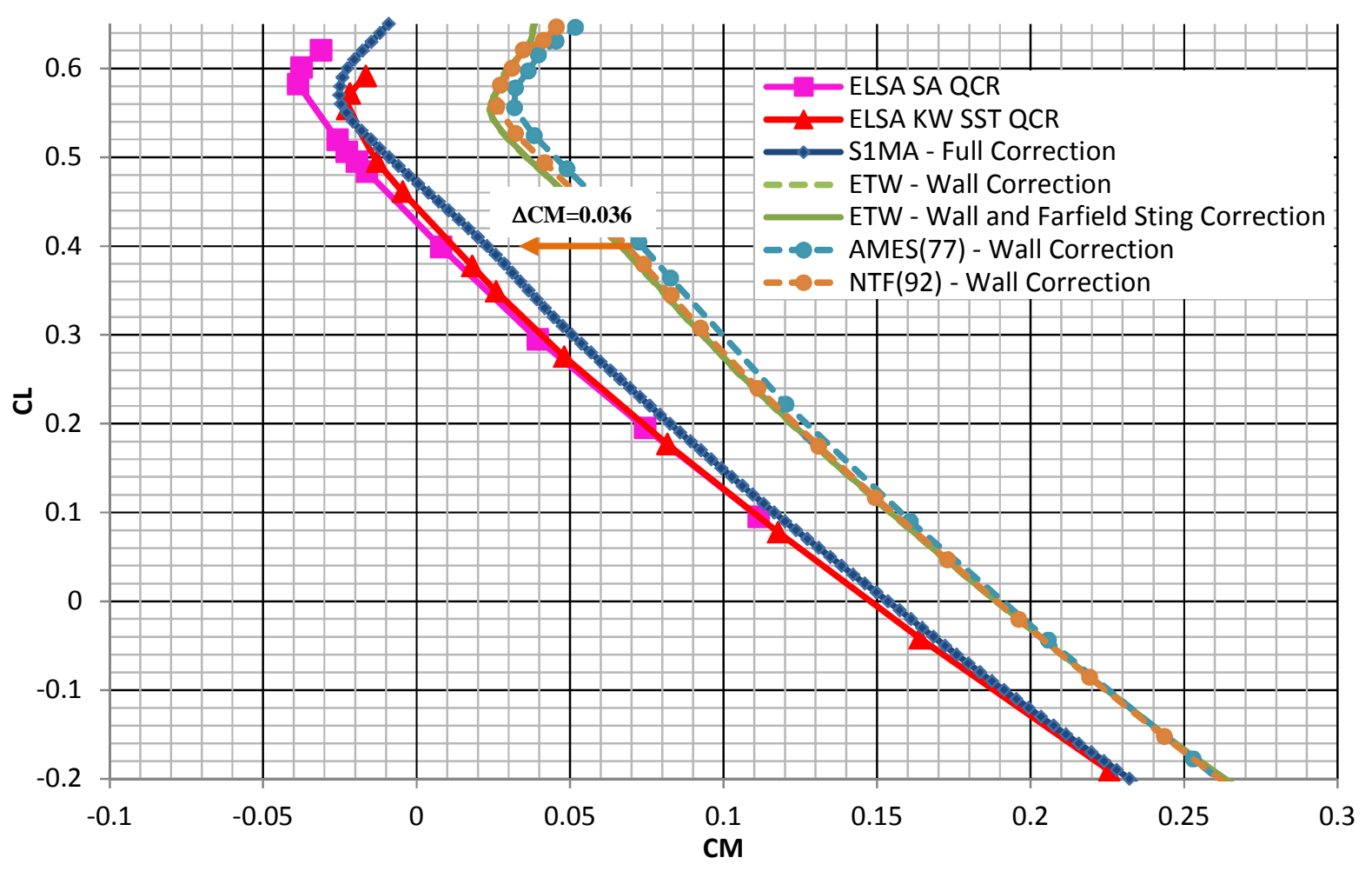

Fig. 36 Pitching moment for $\mathrm{WBH}$ at $M a=0.85$. 


\section{Perspectives about Drag Prediction}

Improvements in both experimental and numerical procedures can be made in order to better compare CFD and wind tunnel drag predictions. It should be said that the computations are fully turbulent whereas the experimental tests are laminar up to $10 \%$ chord, which generates a lower drag. On the other hand, the boundary layer tripping elements which are used on the model should increase the drag. The next step would be to consider the laminar zone and the height and roughness of the trip dots as well as the model roughness itself in order to assess the effects on drag values. Concerning the S1MA experimental results, support effects have been calculated on the full WBVH configuration for which increments on the vertical tail have then been subtracted. Besides, the support effects were calculated on the original DPW-5 geometry (with twist issue unsolved). To achieve a greater consistency, the support corrections will be calculated again but on the WBH configuration with DPW-6 wing geometries so that a better comparison with the latest CFD results can be carried out.

\section{Conclusions}

The article is focused on the comparisons between numerical and experimental results of the CRM-S1MA (LRM) that have been obtained at ONERA.

First, configuration effects (HTP/VTP increments) calculated on the new CRM wing geometry based on twist and bending measurements have been evaluated and compared to experimental results obtained at the ONERAS1MA wind tunnel. The comparison is rather good. The drag increment due to the tails is about 30 counts up to $C L$ $=0.6$.

Afterwards, the flow separation at wing-body junction has been investigated. The CFD data was compared to colored oil flow visualizations performed at cruise point on the WBHV configuration in S1. Grid and turbulence model effects (including QCR) on the flow separation prediction have been highlighted. Good agreement was globally obtained for the flow separation width while its length appeared to be harder to quantify and predict. In this case, the QCR seems to produce flow separations too limited compared to the experiments.

Finally, some absolute comparisons between numerical and experimental pressure, drag and moment data were performed. For this purpose, test results obtained in all four wind tunnels (Ames, NTF, ETW and S1MA) were used. The pressure distributions compare well between all wind tunnels and CFD at cruise point. Nevertheless, the CFD seems to over-predict the aft-loading. At higher lift, experimental results are all in agreement, CFD simulations are in good agreement on the inner wing but discrepancies concerning the shock position appear in the outer sections.

Considering the drag prediction, the turbulence model effect is rather important and the k $\omega$-SST model used in this study happens to give a better agreement than SA with fully corrected experimental results. It has been highlighted that the support effects have to be taken into account both for drag and moment predictions. These effects are considered in S1MA outcomes by means of CFD increments; this allows CFD without support and experimental results to be compared.

Investigations on more adapted support effect corrections and numerical simulations taking into account the boundary layer features are in progress and will be presented in coming articles. 


\section{Acknowledgments}

The authors would like to thank the ONERA-S1MA team. They also thank the DPW Organizing Committee and Melissa Rivers. The numerical studies presented in this article have been performed with the software elsA which is co-owned by AIRBUS, SAFRAN, and ONERA (ASO).

\section{References}

${ }^{1 .}$ Vassberg, J. C., DeHann, M. A., Rivers, S. M., and Wahls, R. A., "Development of a Common Research Model for Applied CFD Validation Studies,” AIAA Paper 2008-6919, 2008.

2.NASA, Langley Research Center, “Common Research Model,” http://commonresearchmodel.larc.nasa.gov/.

${ }^{3}$ DPW-6 website, http://aiaa-dpw.larc.nasa.gov/

${ }^{4}$ Hue, D., and Esquieu, S., "Computational Drag Prediction of the DPW4 Configuration Using the Far-Field Approach," Journal of Aircraft, Vol. 48, No. 5, Sept.-Oct. 2011, pp. 1658-1670.

${ }^{5}$ Hue, D., "Fifth Drag Prediction Workshop: Computational Fluid Dynamics Studies Carried Out at ONERA," Journal of Aircraft, Vol. 51, No. 4, July.-August. 2014, pp. 1295-1310.

${ }^{6}$ Hue, D., "Fifth Drag Prediction Workshop: ONERA Investigations with Experimental Wing Twist and Laminarity," Journal of Aircraft, Vol. 51, No. 4, July.-August. 2014, pp. 1311-1322.

${ }^{7}$ Hue, D., Chanzy, Q., and Landier, S., "DPW-6: Drag Analyses and Increments Using Different Geometries of the CRM Airliner," Journal of Aircraft, published online January 2017.

${ }^{8}$ ONERA website, http://windtunnel.onera.fr/s1ma-continuous-flow-wind-tunnel-atmospheric-mach-005-mach-1

${ }^{9}$ Hue, D., et al., "Validation of a near-body and off-body grid partitioning methodology for aircraft aerodynamic performance prediction," Computers \& Fluids, Vol. 117, 2015, pp. 196-211.

${ }^{10 .}$ Website ESWIRP for ETW data, http://www.eswirp.eu/ETW-TNA-Dissemination.html

${ }^{11 .}$ Le Sant, Y., Mignosi, A., Touron, G., Deléglise, B., Bourguignon, G., "Model Deformation Measurement (MDM) at ONERA", 25th AIAA Applied Aerodynamics Conference, AIAA 2007-3817, Miami, 25-28, June 2007

${ }^{12}$ Vaucheret X., "Recent Calculation Progress on Wall Interferences in Industrial Wind Tunnels", La Recherche Aérospatiale, No. 3, pp 45-47,1988.

${ }^{13}$ Pistolesi, E., "Collected lectures of the principal meeting of the Lilienthal society", Considering respecting the mutual influence of system of airfoils, Berlin, 1937.

${ }^{14}$ Cartieri, A., and Mouton, S., "Using CFD to calculate support interference effects," AIAA Paper 2012-2864, 2012.

${ }^{15 .}$ Rivers M.B., Hunter C.A., Campbell R.L., "Further Investigation of the Support System Effects and Wing Twist on the NASA Common Research Model”, AIAA Paper 2012-3209, 2012.

${ }^{16 .}$ Péron, S., Benoit, C., Landier, S., and Raud, P., "Cassiopée: CFD Advanced Set of Services In an Open Python EnvironmEnt," 12th Symposium on Overset Grid and Solution Technology, Atlanta, 2014.

${ }^{17 .}$ Mesh Generation Software for CFD - Pointwise, http://www.pointwise.com

${ }^{18}$ Cambier, L., Heib, S., and Plot, S., "The ONERA elsA CFD Software: Input from Research and Feedback from Industry," Mechanics and Industry, Vol. 15(3), pp. 159-174, 2013.

${ }^{19}$ Jameson, A., Schmidt, W., and Turkel, E., "Numerical Solution of the Euler Equations by Finite Volume Methods Using Runge Kutta Time Stepping Schemes,” AIAA-81-1259, June 1981. 
${ }^{20 .}$ Spalart, P. R., and Allmaras, S. R., "A One-Equation Turbulence Model for Aerodynamic Flows," AIAA Paper 92-0439, 1992.

${ }^{21 .}$ Menter, F. R., "Two-Equation Eddy-Viscosity Turbulence Models for Engineering Applications," AIAA Journal, Vol. 32, No. 8, August 1994, pp. 1598-1605.

${ }^{22}$ Zheng, X., and Liu, F., "Staggered upwind method for solving Navier-Stokes and k-omega turbulence model equations," AIAA Journal, 1995, 33(6), pp. $991-998$.

${ }^{23 .}$ Wilcox, D. C., "Reassessment of the Scale-Determining Equation for Advanced Turbulence Models," AIAA Journal, Vol. 26, No. 11, 1988, pp. 1299-1310.

${ }^{24 .}$ Spalart, P. R., "Strategies for Turbulence Modelling and Simulation," International Journal of Heat and Fluid Flow, Vol. 21, 2000, pp. 252-263.

${ }^{25}$ DPW-5 website, https://aiaa-dpw.larc.nasa.gov/Workshop5/presentations/DPW5_Presentation_Files/14_DPW SummaryDraft_V7.pdf, pp. 59-61.

26. Zilliac G.G., Pulliam T.H, Rivers M.B., Zerr J., Delgado. M, Halcomb N., Lee H. "A comparison of the Measured and Computed Skin Frcition Distribution on the Common Research Model”, AIAA Paper 2011-1129, 2011.

${ }^{27}$ Rivers M.B., Dittberner A., "Experimental Investigations of the NASA Common Research Model in the NASA Langley National Transonic Facility and NASA Ames 11-Ft Transonic Wind Tunnel (invited)”, AIAA Paper 2011-1126, 2011.

${ }^{28 .}$ Vassberg, et al., "Summary of the Fourth AIAA CFD Drag Prediction Workshop," AIAA Paper 2010-4547, 2010. 